\title{
1 Distinct ribosome states trigger diverse mRNA quality control 2 pathways
}

3

Anthony J. Veltri ${ }^{1}$, Karole N. D'Orazio ${ }^{1+}$, Laura N. Lessen ${ }^{1 \ddagger}$, Raphael Loll-Krippleber ${ }^{2}$, Grant W. Brown ${ }^{2}$, Rachel Green ${ }^{1}$

1. Department of Molecular Biology and Genetics, Howard Hughes Medical Institute, Johns Hopkins University School of Medicine, Baltimore, Maryland, USA.

2. Department of Biochemistry and Donnelly Centre, University of Toronto, Toronto, Ontario, Canada.

${ }^{\dagger}$ Present affiliation: Department of Cell Biology, Harvard Medical School, Boston, Massachusetts, USA

${ }^{\ddagger}$ Present affiliation: GlaxoSmithKline, Rockville, Maryland, USA

\section{Abstract}

Key protein adapters couple translation to mRNA decay on specific classes of problematic mRNAs

in eukaryotes. Slow decoding on non-optimal codons leads to codon-optimality-mediated decay (COMD) and prolonged arrest at stall sites leads to no-go decay (NGD). The identities of the decay factors underlying these processes and the mechanisms by which they respond to translational distress remain open areas of investigation. We use carefully-designed reporter mRNAs to perform genetic screens and functional assays in S. cerevisiae. We characterize the roles of Hel2 and Syh1 in coordinating translational repression and mRNA decay on NGD reporter mRNAs, finding that Syh1 acts as the primary link to mRNA decay in NGD. Importantly, we observe that these NGD factors are not involved in the degradation of mRNAs enriched in non-optimal codons. Further, we establish that a key factor previously implicated in COMD, Not5, contributes modestly to the degradation of an NGD-targeted mRNA. Finally, we use ribosome profiling to reveal distinct ribosomal states associated with each reporter mRNA that readily rationalize the contributions of NGD and COMD factors to degradation of these reporters. Taken together, these results provide new mechanistic insight into the role of Syh1 in NGD and define the molecular triggers that determine how distinct pathways target mRNAs for degradation in yeast. 


\section{Introduction}

Translation of mRNAs to produce proteins is a fundamental cellular process that supports the cell's ability to carry out the basic enzymatic reactions needed for life. To prevent errors that arise during this complex process from compromising cellular metabolism, specialized molecular pathways have evolved to recognize and regulate problematic translation events (D'Orazio and Green 2021; Inada 2017; Yan and Zaher 2019). These mechanisms are coupled to RNA decay pathways that target problematic mRNAs and prevent continuing diversion of ribosomes toward unproductive translation. The set of factors involved in this crucial recognition of problems that arise during translation elongation and the mechanisms by which they exert their downstream effects on mRNA stability remain only partially characterized.

General mRNA decay in yeast is catalyzed primarily by mRNA decapping and 5' to 3' exonucleolytic degradation by Xrn1, while the $3^{\prime}$ to $5^{\prime}$ exonuclease (the exosome) is thought to play a role only under certain circumstances (Muhlrad, Decker, and Parker 1994). Recent foundational work in yeast, and subsequently in zebrafish and mammals, discovered that mRNA stability is correlated with its codon usage (Presnyak et al. 2015; Mishima and Tomari 2016; Q. Wu et al. 2019): mRNAs enriched in non-optimal codons have short half-lives and are rapidly degraded by the cytoplasmic Ccr4-Not deadenylation complex and the decapping activator Dhh1 (Radhakrishnan et al. 2016; Webster et al. 2018; Sweet, Kovalak, and Coller 2012). Recent biochemical and structural evidence supports a model in which suboptimal codons

44 in the ribosomal A site slow down translation elongation, allowing deacylated tRNA to diffuse away from the $\mathrm{E}$ site and enabling the critical adaptor protein Not5 to bind. Not5 binding in the vacant ribosomal $\mathrm{E}$ sites can recruit the Ccr4-Not complex to promote mRNA deadenylation, decapping, and decay

47 (Buschauer et al. 2020). These observations provide molecular insight into codon-optimality-mediated decay (COMD) and support for the idea that this pathway represents an important determinant of general 
In contrast to the normal slowing of translation that occurs transiently as ribosomes decode less

51

optimal codons, the cell also possesses quality-control machinery to resolve more deleterious ribosomal stalls that can arise from chemical damage in the mRNA (truncation, depurination, nucleobase dimers, oxidative damage, etc.), difficult to unwind secondary structure, or incorrect nuclear mRNA processing events (D'Orazio and Green 2021). Adjacent pairs of specific rare codons can mimic these events and induce strong inhibition of translation elongation and associated mRNA decay in S. cerevisiae (Gamble et al. 2016). For example, consecutive CGA codons induce terminal stalls and have been routinely included in reporter mRNAs to trigger an mRNA surveillance pathway referred to as no-go decay (NGD) (Tsuboi et al. 2012; Letzring et al. 2013; Tesina et al. 2020). On these problematic mRNAs, ribosomes stall on the CGA codons, leading to ribosomal collisions that promote small-subunit protein ubiquitination by the E3 ligase Hel2 (mammalian ZNF598), ribosomal clearance by the helicase SIh1 and ribosome quality control trigger (RQT) complex (Ikeuchi, Izawa, and Inada 2019), and nascent peptide decay by the ribosome quality control (RQC) complex (Brandman et al. 2012). The accumulation of colliding ribosomes is thought to trigger decapping and Xrn1-mediated mRNA degradation, though the specific molecular players and interactions responsible for triggering this decay remain poorly defined (D'Orazio et al. 2019; Simms et al. 2019). Additionally, under conditions where the ribosome rescue machinery is compromised or overwhelmed, cleavage of reporter mRNAs by the endonuclease Cue2 and Dom34-mediated rescue provides an alternate route for mRNA degradation and ribosome rescue (Doma and Parker 2006; D'Orazio et al. 2019; Glover et al. 2020). NGD, as a "quality control" pathway, is thought to minimize the detrimental effects from damaged or problematic mRNAs in the cell and to reduce the overall impact of proteotoxic stress (Brandman et al. 2012; Ishimura et al. 2014; Martin et al. 2020).

Though NGD and COMD can be triggered by seemingly similar mRNA sequences and converge on Xrn1-mediated exonucleolytic decay of mRNAs (Pelechano, Wei, and Steinmetz 2015), the extent to which these processes overlap in specificity and activity remains unclear, as do their complete sets of accessory 
74 factors. Moreover, the molecular states of the ribosome which define and activate these pathways have

75 not been systematically compared. In this study, we address these questions through genetic screening and functional assays in the yeast S. cerevisiae. We use reporter mRNAs designed to trigger NGD or COMD

77 and perform synthetic genetic array (SGA) screens to identify factors critical to these separate mRNA

78 decay pathways. Importantly, we identify a critical role for Syh1 as the primary effector for decay for

79 mRNAs with terminal stalls. We use flow cytometry and northern blotting combined with genetic

80 perturbations to reveal the contributions of other NGD factors and COMD factors to translational

81 repression and decay of the reporter mRNAs. Finally, we use ribosome profiling of NGD and COMD

82 reporters to isolate the activities of the major players in these pathways and connect these activities to

83 the molecular states of elongating ribosomes. These data provide a basis for understanding the unique

84 contributions of each of these pathways to translation-coupled mRNA decay and contextualizes their

85 effects in the larger cellular process of translation surveillance.

\section{Results}

\section{A genetic screen identifies NGD factors in yeast}

To identify protein factors that contribute to NGD, we developed reporter constructs with welldefined sequence features designed to trigger ribosome stalling and associated quality control. Reporter mRNAs were under the control of the inducible, bidirectional GAL1-10 promoter and encoded GFP

91 followed by either a fully codon-optimized yeast HIS3 gene (termed OPT) or HIS3 interrupted by twelve

92 repeats of the highly non-optimal CGA codon (termed CGA; Figure 1A). This repeat sequence has been

93 shown to trigger NGD in S. cerevisiae by causing strong stalling of ribosomal elongation (due to overall

94 low abundance of tRNA ${ }^{\text {Arg(ICG) }}$ compounded by inefficient decoding by the I:U wobble interaction) and 95 ensuing ribosome collisions (Letzring, Dean, and Grayhack 2010; Tesina et al. 2020). A viral P2A sequence 
bioRxiv preprint doi: https://doi.org/10.1101/2021.12.01.470814; this version posted December 1, 2021. The copyright holder for this preprint (which was not certified by peer review) is the author/funder, who has granted bioRxiv a license to display the preprint in perpetuity. It is made

A)

B)
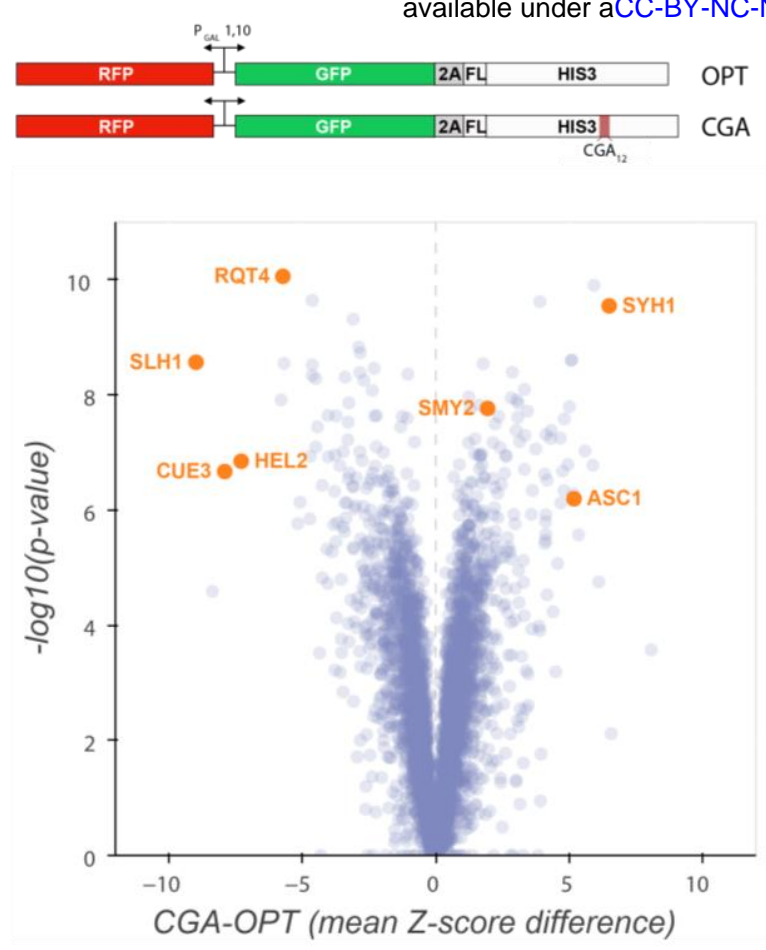

E)

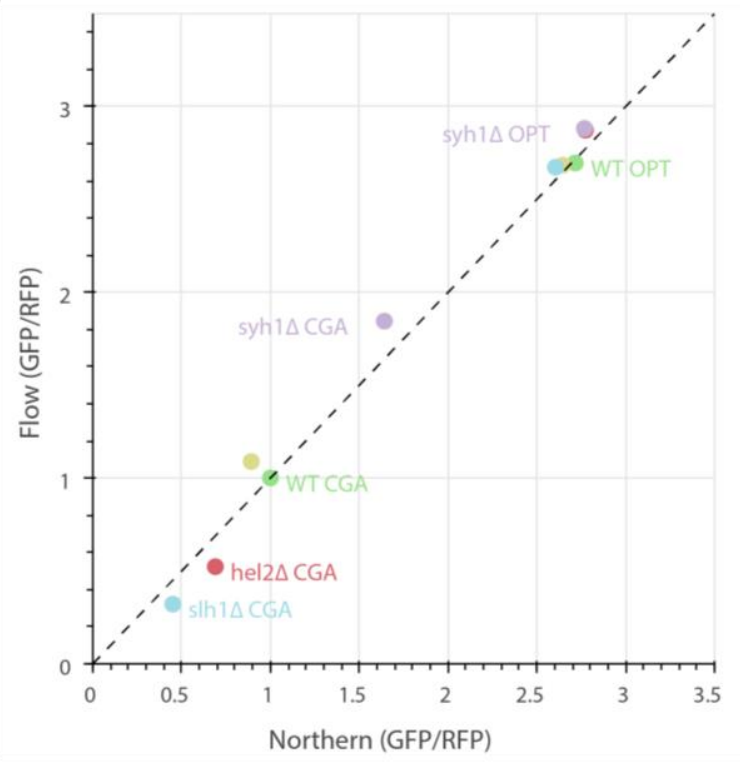

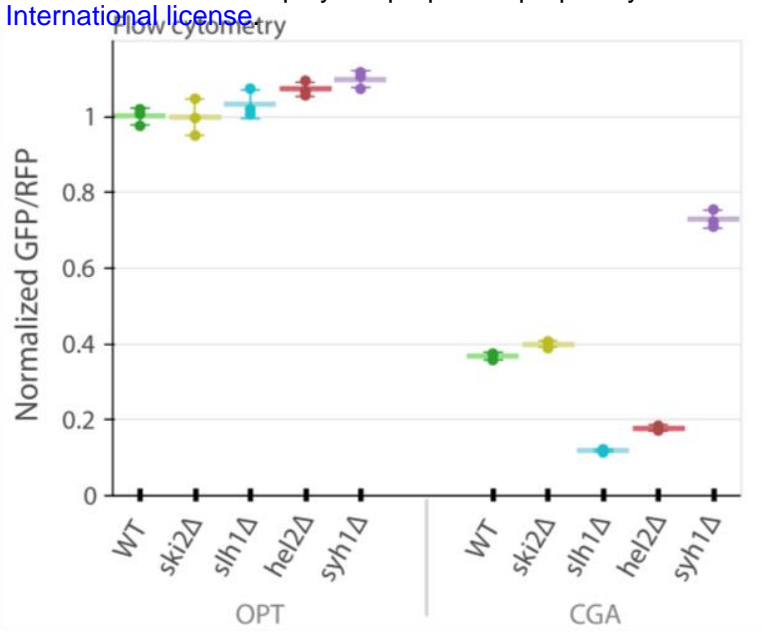

D)
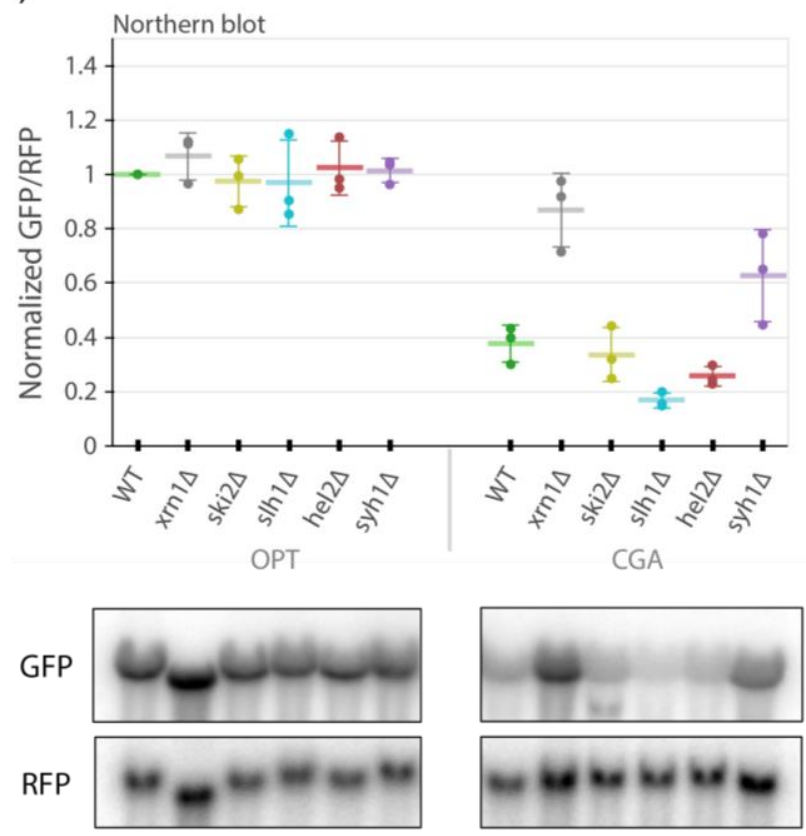

Figure 1: A genetic screen reveals factors that alter levels of an NGD reporter

A) Diagrams of OPT and CGA reporters. Reporters are expressed from a bidirectional GAL promoter. GFP is separated from the HIS3 ORF by a P2A "StopGo" sequence. The CGA reporter contains an insert of twelve CGA codons as a stalling sequence.

B) Volcano plot of data from the R-SGA screen. P-values were calculated from a Fisher's t-test based on per plate GFP/RFP Z-score differences between the CGA and OPT screens. Positive Z-score differences indicate an increase of the CGA reporter relative to the OPT reporter and vice versa. Selected genes are labeled.

C) Flow cytometry analysis of OPT and CGA GFP protein fluorescence reporter levels normalized to RFP fluorescence in several genetic backgrounds. All GFP/RFP levels are normalized to the mean of WT OPT and three replicates are plotted for each strain. Error bars indicate standard deviation.

D) Northern blot analysis of OPT and CGA GFP mRNA reporter levels normalized to RFP mRNA levels quantified by probe hybridization and autoradiography. Three replicates are plotted for each background. All GFP/RFP levels are normalized to the level of WT OPT within each replicate set. Error bars indicate standard deviation. Representative images of the northern blots for one replicate set are shown.

E) The mean reporter levels from panels $C$ and $D$ were normalized and replotted to allow comparison between flow cytometry and northern blot results. All data within each assay were normalized to the mean WT CGA reporter levels. 
(Brown and Ryan 2010; Sharma et al. 2012) was inserted between the GFP and HIS3 open reading frames

97 to decouple GFP levels from the protein decay induced by RQC factors in response to ribosome pausing on CGA codons. Importantly, knockout of LTN1, the major E3 ligase responsible for nascent peptide degradation by the RQC complex, did not increase the GFP/RFP ratio for our reporter (Figure S1A), demonstrating that nascent peptide decay does impact the levels of GFP protein. As a result, for this construct, GFP levels serve as a proxy for reporter mRNA levels and translation initiation rates, allowing us to follow these activities in individual cells by flow cytometry. An RFP mRNA is produced from the same GAL1-10 promoter in the reverse direction, allowing RFP fluorescence to be used to normalize for average transcription and metabolic changes within individual cells. introducing the OPT and CGA reporters into the 5377 yeast strains contained in the Yeast Knockout

107 Collection (Giaever et al. 2002). A total of 4222 deletion strains were successfully grown and tested with fluorimetry. We obtained GFP and RFP data for each deletion strain with these two reporters and calculated Z-scores of the GFP/RFP ratio for every strain on a per-plate basis, allowing comparison between the CGA screen and the previously published OPT screen (Figures 1B, S1B) (D'Orazio et al. 2021).

111 To identify genes contributing to NGD, we focused on knockout strains in which normalized CGA reporter 112 levels were significantly increased or decreased relative to our normalized OPT reporter levels 113 (Supplementary Table 1, Figures 1B, S1B). Among the strongest hits from the screen were known NGD 114 factors including HEL2 and members of the RQT complex (SLH1, CUE3, and RQT4), all of which exhibited 115 substantially decreased GFP reporter fluorescence compared to the wild-type control. This suggests that 116 loss of these factors causes increased decay of the reporter mRNA. Among the strongest hits that 117 increased CGA reporter levels, we identified the ribosomal protein gene ASC1 and the genes SYH1 and SMY2, homologs of the mammalian NGD factors GIGYF1/2, which we previously reported to impact CGA 
identical to the CGA reporter, except with the $\mathrm{CGA}_{12}$ repeat replaced by $A A A_{12}$; results from this screen

121 showed broad overlap with the CGA reporter screen (Figures S1C-D) as anticipated based on the similar

122 stalling mechanisms of these sequences (Tesina et al. 2020; Koutmou et al. 2015).

Of the CGA reporter strains tested in the original screen, we selected 170 with the strongest

increases or decreases in GFP levels (-2 > CGA Z-score $>2$ and -2 < OPT Z-score < 2) and individually

validated them by flow cytometry to determine which backgrounds affected CGA reporter levels. As in

strongly impacted GFP levels, validating our screen results and providing confidence for further

(Supplementary Table 3, Figure S1F).

\section{Functional assays recapitulate known effects of NGD Factors}

To further explore the hits from our genome-wide screen, we deleted genes of interest to verify

the observed effects in a clean knockout background and to establish the mechanism of repression for

134 the CGA reporter. We integrated the OPT and CGA reporters at the ADE2 locus in yeast strains with deletions of SYH1 and other factors including HEL2, SLH1, SKI2 and XRN1. While our screen identified two homologs of mammalian GIGYF1/2, our earlier studies had shown that stronger effects were associated

137 with deletion of SYH1 than with SMY2 (Hickey et al. 2020), therefore for simplicity, we focused here on 138 the SYH1 deletion strain. In flow cytometry experiments, we found that the GFP/RFP fluorescence ratios for the OPT reporter were not strongly affected by deletion of HEL2, SLH1, SKI2 or SYH1 (Figure 1C). In the wild-type strain, CGA reporter levels were reduced $\sim 3$-fold in comparison to OPT as expected for a 

reporter levels. unaltered across all the deletions strains and the CGA mRNA reporter level was reduced relative to the OPT mRNA reporter level in the wild-type strain (Figure 1D). In agreement with the flow cytometry data and previous literature, ski2 $\Delta$ had no effect on CGA reporter levels, whereas xrn1 $\Delta$ strongly rescued reporter levels (D'Orazio et al. 2019; Simms et al. 2019). These results are consistent with the long established importance of Xrn1 in general mRNA decay (Muhlrad, Decker, and Parker 1994) and its critical role in NGD (D'Orazio et al. 2019). Other patterns observed in the flow cytometry data were recapitulated here as well: reporter levels were decreased to a similar extent in the hel2 $\Delta$ and $\operatorname{sih} 1 \Delta$ strains as in flow cytometry (Figure 1E). And, as revealed by our screen, syh1 $1 \Delta$ substantially rescued mRNA reporter levels. This focused analysis of protein and RNA reporter levels in these different strains validates results from the initial screen and indicates that the CGA reporter is being strongly regulated by canonical NGD 157 machinery.

\section{Syh1 is the primary NGD factor in yeast}

Since screens in this study and our previous work (D'Orazio, et al. 2019) implicated HEL2, CUE2, and SLH1 in altering CGA reporter levels, we asked how knockouts of these factors would alter the reporter mRNA levels in an syh1 $\Delta$ strain to get some indication about epistasis. In order to compare more systematically the effects of these knockouts, we turned to a simpler set of reporters expressed from plasmids and containing problematic sequences within the HIS3 gene but lacking the upstream GFP ORF and the P2A sequence (Figure $2 \mathrm{~A}$ ). The P2A sequence, in particular, has been shown to induce ribosome 
bioRxiv preprint doi: https://doi.org/10.1101/2021.12.01.470814; this version posted December 1, 2021. The copyright holder for this preprint (which was not certified by peer review) is the author/funder, who has granted bioRxiv a license to display the preprint in perpetuity. It is made

A) available under aCC-BY-NC-ND 4.0 International license.

\begin{tabular}{|l|l|l|l|}
\hline FL & HIS3 & HIS3 & minCGA \\
CGA $_{12}$ & minOPT
\end{tabular}

B)

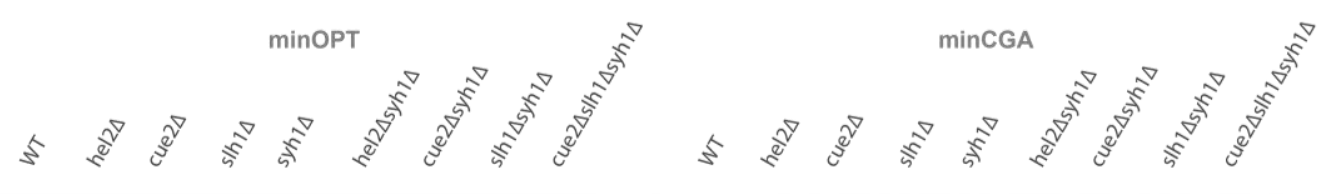

HIS3
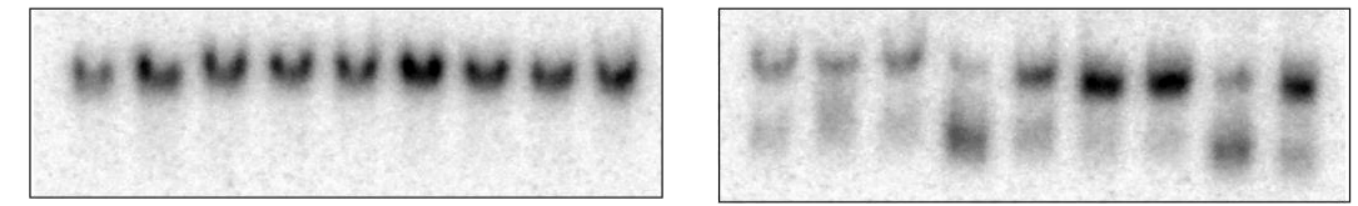

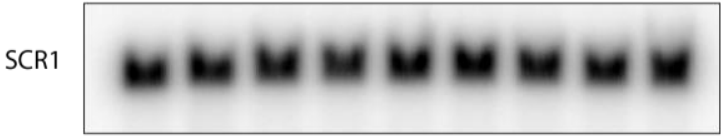

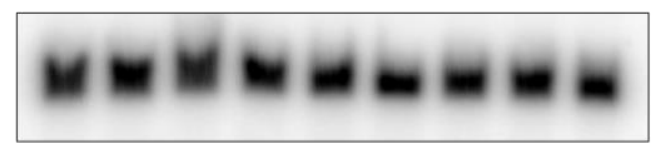

C) Full length

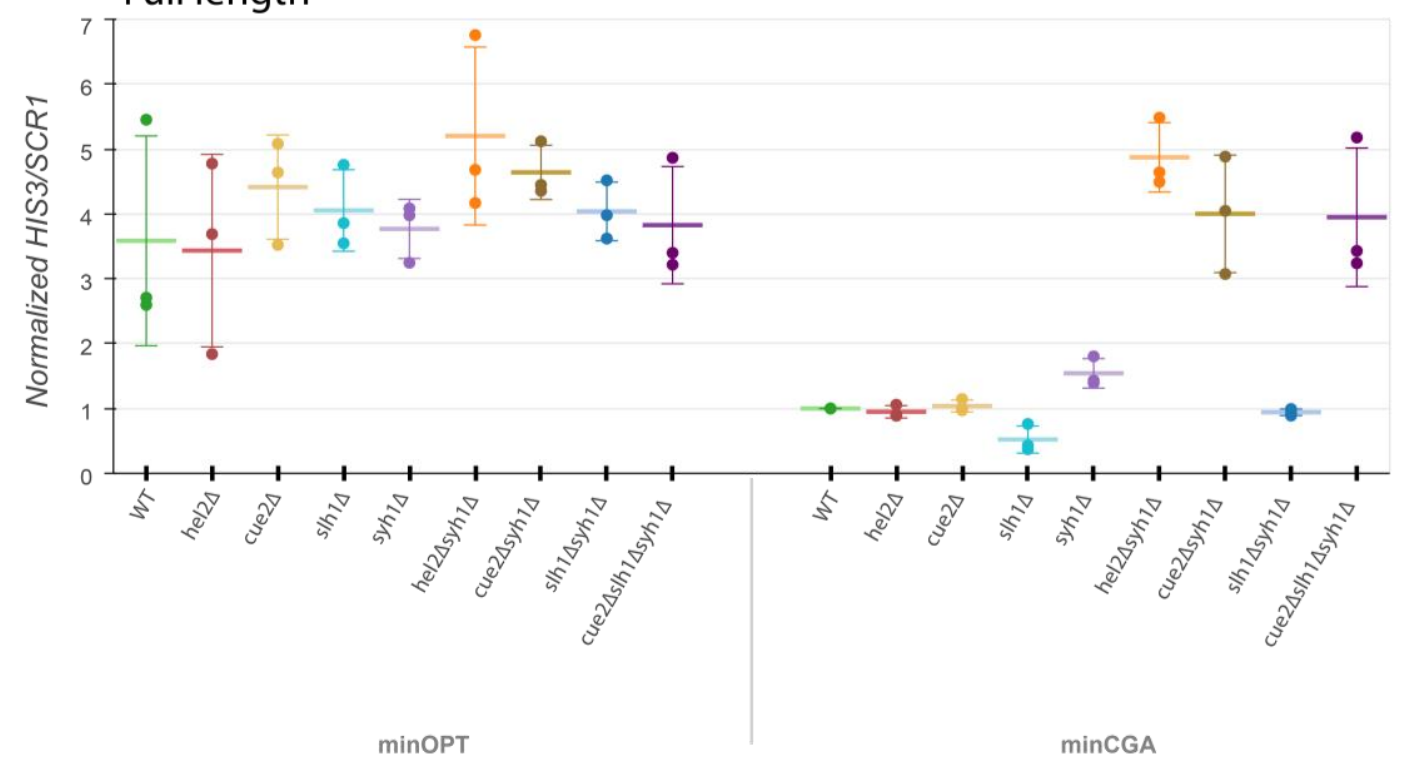

Figure 2: Syh1 is a critical mRNA decay factor for an NGD reporter

A) Diagrams of minOPT and minCGA reporters. Reporters are expressed from a GAL promoter and contain only a FLAG tag and HIS3 ORF. The minOPT reporter contains a fully-optimized ORF, the minCGA reporter contains a CGA $_{12}$ stalling sequence within the ORF as indicated by red shading.

B) Autoradiograph of northern blot of one representative replicate set of reporter mRNA levels for strains and probes as indicated.

C) Northern blot quantification of minOPT and minCGA HIS3 mRNA reporter levels normalized to SCR1 mRNA levels quantified by probe hybridization and autoradiography in yeast strains containing various NGD factor knockouts. Three replicates are plotted. All HIS3/SCR1 RNA levels are normalized to the levels of WT CGA RNA within each replicate set. Error bars indicate standard deviation. 
2020). The minOPT reporter is a fully codon optimized, N-terminally FLAG-tagged HIS3 sequence expressed under a GAL promoter, and the minCGA reporter is identical to the minOPT reporter except it includes twelve CGA repeats in the same codon position within HIS3 as in the GFP-containing CGA reporter used in screening.

To recapitulate our findings with the new minCGA stalling reporter, we used northern blotting to assess the steady-state levels of the reporter mRNA (Figures 2B-C). First, hel2 $\Delta$ does not decrease minCGA reporter levels as observed with the GFP-containing CGA reporter; this discrepancy may arise from effects

173 of the P2A sequence or from differences in levels of expression and thus in ribosome loading. Importantly,

174 although the loss of CUE2 alone has little effect on the minCGA reporter, the loss of SLH1 results in a 175 decrease in the amount of full-length mRNA and a corresponding accumulation of a 3' reporter fragment 176 (Figure 2B, HIS3 probe bottom band; Figures 2C, S2A). This is consistent with our previous studies that 177 established that NGD proceeds through Xrn1 under normal circumstances, whereas the endonuclease 178 Cue2 plays a more important role in the absence of $S L H 1$ (D'Orazio et al. 2019). In this paradigm, Cue2 acts a failsafe that only cuts mRNAs when stalled ribosomes accumulate when Slh1 is absent or overwhelmed. Finally, here again we observe a modest rescue of reporter levels in the syh1 $\Delta$ strain, consistent with a model where Syh1 recruits Xrn1 and the machinery to mediate decay of the CGA reporter. relevant factors and then performed northern blot analysis to follow the levels of the minCGA reporter mRNA. Strikingly, we observe that the hel2 $\Delta$ syh1 $1 \Delta$ strain shows a complete rescue of the minCGA mRNA to the levels of the minOPT reporter (Figure 2C). A similar strong rescue was seen with the GFP-containing 187 CGA reporter (Figure S2B). Because Hel2 functions upstream of other NGD factors in its role as an E3 ubiquitin ligase marking colliding ribosomes (Brandman et al. 2012; Saito, Horikawa, and Ito 2015; Matsuo 
effects on mRNA decay. We found that the cue2 $\Delta$ syh1 $1 \Delta$ strain also showed much higher levels of minCGA reporter, arguing that Cue2 cleavage (which requires Hel2 activity) is the downstream step that degrades mRNA in the absence of Syh1. Further deletion of SLH1 had little or no additive effect in the cue2 $\triangle$ syh1 $\triangle$ strain. These results indicate that Syh1 is the major factor contributing to NGD in the absence of HEL2, but that Cue2 plays a major compensatory role in decay when Slh1 or Syh1 activity is impaired.

Mbf1, a protein previously implicated in ribosome-mediated quality control pathways (Hendrick et al. spectrometry (AP-MS) to search for binding interactions that could help explain the mechanism of action the $m b f 1 \Delta$ strain, suggesting that in S. cerevisiae this factor is not critical for RNA decay mediated by Syh1 (Figure S2D). translation repressor thought to have a similar mechanism of action to the GIGYF2-associated mammalian protein 4EHP (Sezen, Seedorf, and Schiebel 2009; Cosentino et al. 2000) which is known to work together with GIGYF1/2 to bring about translational repression (Morita et al. 2012; Peter et al. 2019). Again, CGA

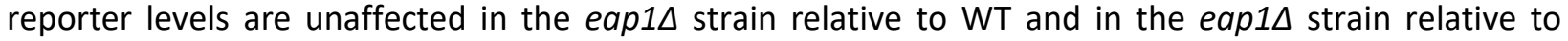

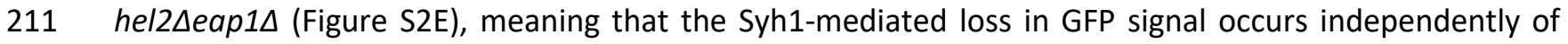


suggest that Syh1 recruitment and function in S. cerevisiae differs substantially from that observed for

214 GIGYF1/2 in mammalian cells.

\section{COMD does not require canonical NGD factors}

Given that the CGA reporter simply contains a stretch of highly non-optimal codons, we wondered

217 whether the same set of factors might similarly regulate ORF sequences containing more widely

218 distributed non-optimal codons. We tested this possibility first by developing a reporter similar to that

219 used for the NGD screen with an N-terminal GFP, an internal P2A sequence, and a downstream HIS3 gene

220 with an internal stretch of 129 codons synonymously re-coded as non-optimal (NONOPT; Figure 3A). As

221 expected, the NONOPT reporter exhibited substantially diminished GFP levels compared to the OPT

222 reporter and thus provided a starting point for subsequent analysis (Figure 3B).

To identify potential contributing factors to COMD, we performed an R-SGA screen (as above)

224 with this NONOPT reporter (Figure 3C) and again compared these results to our OPT screen results.

225 Strikingly, none of the known NGD factors that we had identified in the previous NGD-CGA screen

226 emerged. These data provide a first indication that NGD mRNAs are regulated very differently from non-

227 optimal mRNAs at the molecular level. Interestingly, factors previously implicated in stabilizing non-

228 optimally coded mRNAs (Webster et al. 2018; Radhakrishnan et al. 2016; Buschauer et al. 2020) were also

229 not among the strains that revealed increases in NONOPT reporter levels (Figure 3C). While some of these

230 strains are not present in the deletion strain collection due to their severe growth phenotype ( $d h h 1 \Delta$ and

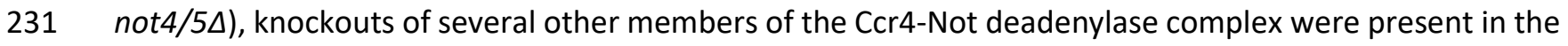

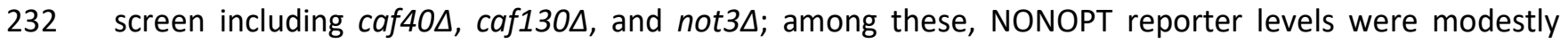

233 increased only in the caf40 strain. 
bioRxiv preprint doi: https://doi.org/10.1101/2021.12.01.470814; this version posted December 1, 2021. The copyright holder for this preprint (which was not certified by peer review) is the author/funder, who has granted bioRxiv a license to display the preprint in perpetuity. It is made available under aCC-BY-NC-ND 4.0 International license.

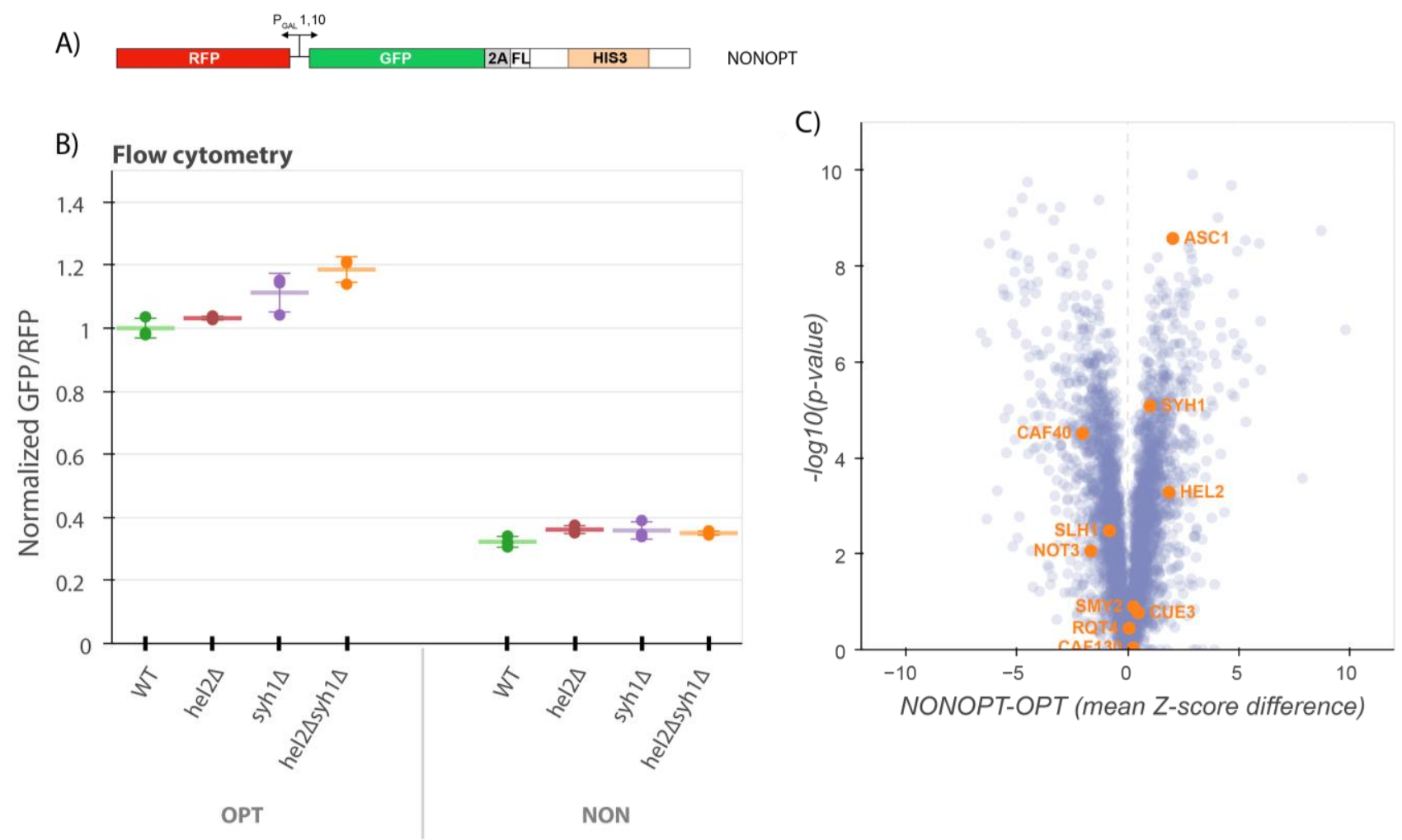

Figure 3: NGD factors do not alter levels of a COMD reporter

A) Diagram of the NONOPT reporter for SGA screening and flow cytometry. Reporter is expressed from a bidirectional GAL promoter. GFP is separated from the HIS3 ORF by a P2A "StopGo" sequence. A portion of the HIS3 ORF is recoded as synonymous codons with low optimality.

B) Flow cytometry analysis of OPT and NONOPT GFP protein fluorescence reporter levels normalized to RFP fluorescence in yeast strains containing knockouts of HEL2 and SYH1 individually and in combination. All GFP/RFP levels are normalized to the mean levels of WT OPT in triplicate. Error bars indicate standard deviation.

C) Volcano plot of the NONOPT R-SGA screen P-values were calculated from a Fisher's t-test based on per plate GFP/RFP Zscore differences between the NONOPT and OPT screens. Positive Z-score differences indicate an increase of the NONOPT reporter relative to the OPT reporter and vice versa. Selected genes are labeled. 
Together, these data suggest that there are fundamental differences in recognition by the decay

237 machinery of overall non-optimal coding sequences and more problematic strong translational stalls.

\section{Exploring differences between non-optimal and NGD-triggering mRNAs}

In order to compare more systematically the effects of mRNA sequences that trigger NGD or

244 (Figure 4A). To evaluate these new reporters, we employed a northern-blot-based transcriptional shutoff assay to measure RNA half-lives as previously reported (Radhakrishnan et al. 2016) in various deletion strains including those implicated above in NGD as well as those previously implicated in COMD. SYH1) had any discernible effect on the stability of the minOPT reporter mRNA (Figures 4B, S4A-B, effect while NOT5 deletion stabilized this mRNA by approximately 11 -fold, consistent with earlier reports

253 (Buschauer et al. 2020). 


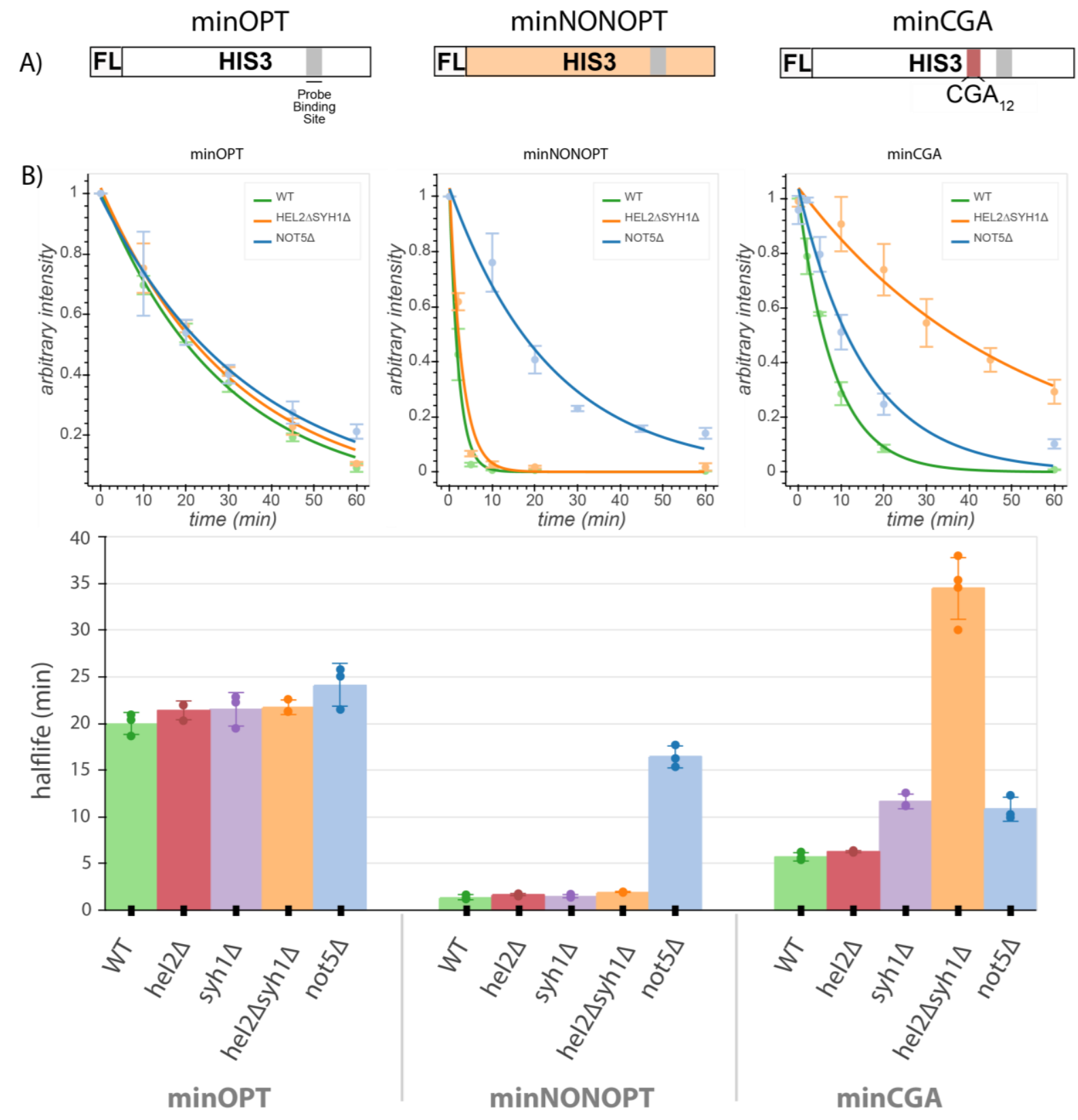

Figure 4: Minimal reporter mRNA half-lives are affected by COMD and NGD factor knockouts

A) Diagram of the minOPT, minCGA, and minNONOPT reporters. Reporters are expressed from a GAL promoter and contain only a FLAG tag and HIS3 ORF. The minOPT reporter contains a fully-optimized ORF, the minCGA reporter contains a CGA 12 stalling sequence within the ORF (indicated by the red shaded region), and the minNONOPT reporter contains a HIS3 ORF fully recoded as synonymous non-optimal codons. All reporters share a common probe binding region for northern blot analysis (shaded in gray).

B) Reporter mRNA half-lives in distinct genetic backgrounds were measured following transcriptional shut down. Reporter mRNA levels were normalized to endogenous SCR1 levels. Top, reporter mRNA decay curves measured by northern blot analysis after GAL promoter shutoff in different genetic backgrounds. A single exponential decay was fit to means of three or four replicates at each timepoint. Bottom, half-lives were calculated for replicates individually by fitting to a single exponential decay and averaged. All error bars indicate standard deviation. 
surprisingly, we find that deletion of NOT5 increases CGA reporter half-life ( 2 -fold), suggesting features

of general non-optimality for this reporter mRNA. Together, these data are broadly consistent with the existence of multiple modes for recognition of troubled elongating ribosomes, each recognized by a distinct set of factors, and leading to decapping and Xrn1-mediated exonucleolytic decay.

\section{Ribosome conformations drive distinct mRNA decay pathways}

With the goal of connecting ribosome states to downstream consequences, we next employed ribosome footprint profiling (Ribo-seq) to characterize the positions and conformational states of elongating ribosomes on the minimal reporter mRNAs (Ingolia et al. 2009). To increase the resolution of

267 the approach, both cycloheximide and tigecycline, two specific elongation inhibitors, were added to lysates to capture distinct rotational states of the ribosome during translation represented by two populations of ribosome protected fragment (RPF) lengths centered at approximately 21 and 28

270 nucleotides (C. C.-C. Wu et al. 2019). The 21-mer population captures ribosomes without A-site tRNAs

271 (waiting to decode) while the 28-mer population captures ribosomes with filled A sites (waiting to 272 translocate). Finally, to further increase the resolution of the study, we separately sequenced single273 ribosome (monosome) footprints and those from nuclease-resistant disomes, thought to represent the 274 collided structure that triggers NGD (Guydosh and Green 2014).

276 are relatively evenly distributed across the entire ORF in the minOPT reporter for both the 21-mer and

277 28-mer tracks (Figure 5A). As previously observed for the GFP-containing CGA reporter (Sitron, Park, and

278 Brandman 2017; D'Orazio et al. 2019), we observed that the CGA stall region profoundly disrupts

279 translation of the minCGA ORF. The density of monosome ribosome footprints (both 28- and 21-mers)

280 downstream of the CGA stall is greatly reduced compared to the upstream region on this reporter, 
bioRxiv preprint doi: https://doi.org/10.1101/2021.12.01.470814; this version posted December 1, 2021. The copyright holder for this preprint (which was not certified by peer review) is the author/funder, who has granted bioRxiv a license to display the preprint in perpetuity. It is made available under aCC-BY-NC-ND 4.0 International license.
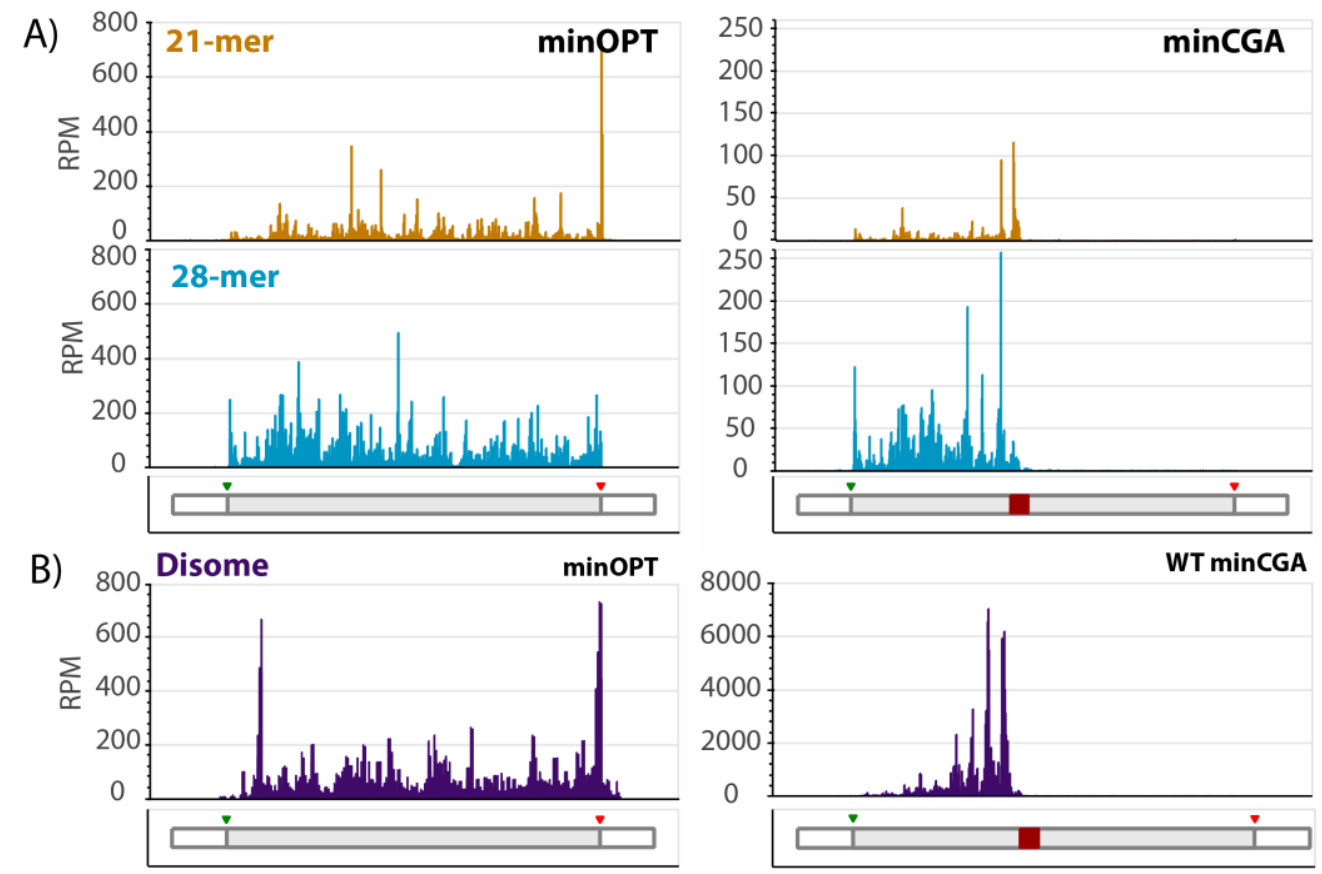

C)

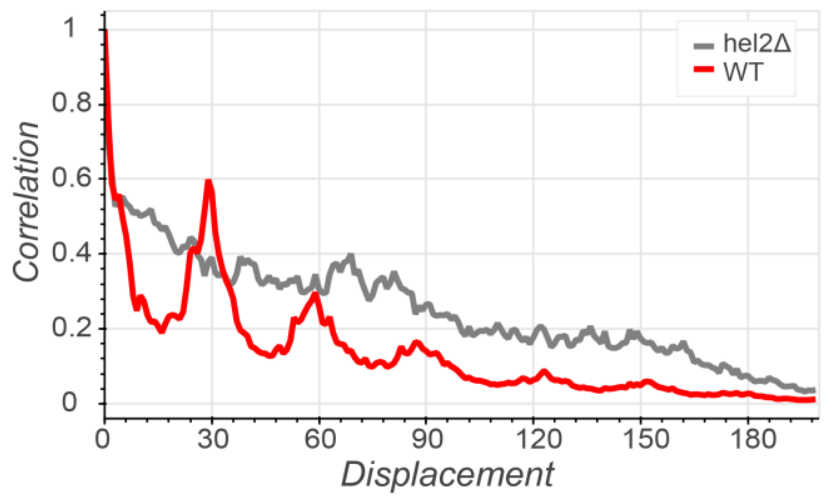

D)

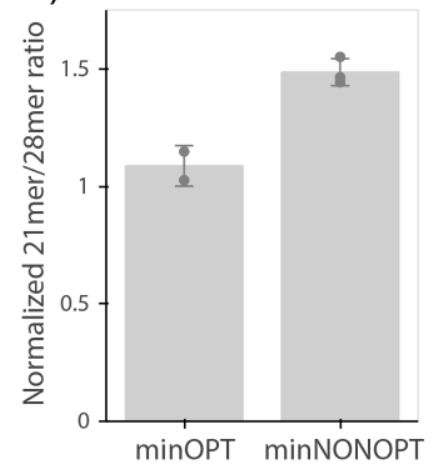

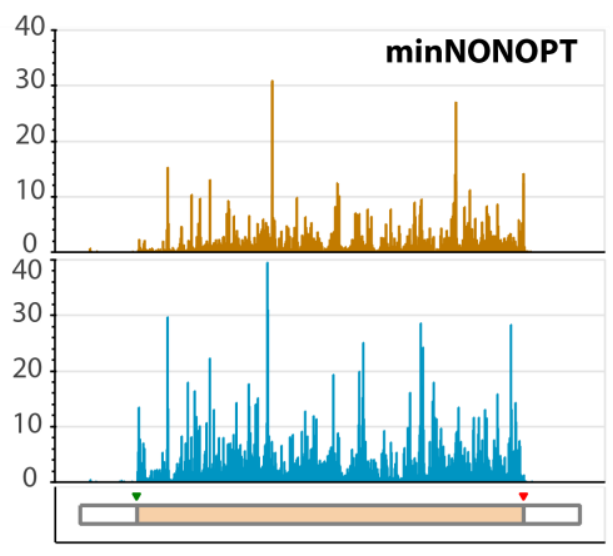

minNONOPT

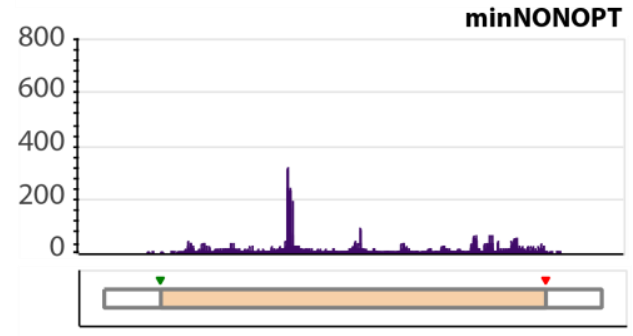

E)

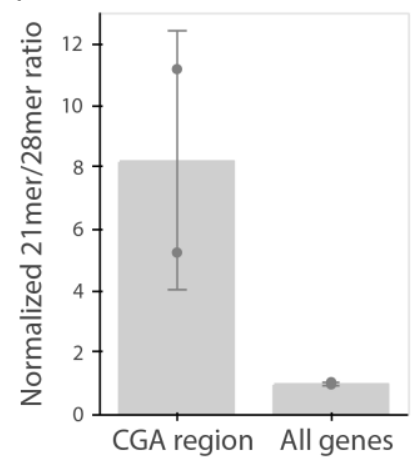

Figure 5: Ribosome profiling reveals translational states that trigger NGD and COMD

A) Gene diagrams show reads per million (RPM) at every position of the indicated reporters: minOPT (left), minCGA (center), minNONOPT (right). Top, 21-mer reads are plotted using read lengths 19-26 inclusive. Bottom, 28-mer reads are plotted using read lengths 27-36 inclusive. Beneath each plot, diagrams of the indicated reporter show the locations of important features.

B) Read RPMs from disome footprint profiling are shown for each position of the minOPT (top) and minNONOPT (bottom) reporters.

C) Autocorrelation of the disome footprint RPMs for WT minCGA and hel2 $\triangle$ minCGA samples.

D) Normalized 21-mer to 28-mer ratios of two replicates for the minOPT and minNONOPT reporters. Ratios exclude regions near the start and end of the ORF and the common probe binding region and are normalized to the 21-mer to 28-mer ratio of all genes to control for differences in digestion efficiency between libraries.

E) Normalized 21-mer to 28-mer ratios of two replicates for multiply aligned reads within the CGA region of the minCGA reporter. Ratios are normalized to the average of 21-mer to 28-mer ratios for all genes. 
S5B, top). Importantly, there is a high density of 21-mers near the beginning of the CGA repeat region, consistent with previous reports that decoding of CGA codons is slow. Additionally, 21 and 28-mer peaks appear approximately one footprint length upstream of the CGA stall, extending backwards in a repeating pattern approximately every ribosome length as confirmed by autocorrelation analysis (Figure S5A, S5C top row). region upstream of the CGA codons; and, autocorrelation analysis reveals that the pattern of "stacked" disomes is periodic with peaks at approximately 30 nucleotide intervals (Figure 5B, center; Figure 5C). In this analysis, disome RPM peaks on the minCGA reporter are dramatically increased ( 10 -fold) relative to the minOPT reporter, indicative of an accumulation of collided ribosomes (compare scales of minOPT and minCGA panels in Figure 5B). These data together suggest that a key signal for the recruitment of Syh1 and the NGD machinery is the collided ribosome.

To further explore a potential ribosomal basis for the activity of NGD factors on stalling reporter levels, we performed ribosome profiling on the minCGA reporter in hel2 $\Delta$ and syh1 $1 \Delta$ strains. First, as previously observed (Letzring et al. 2013), deletion of HEL2 increased ribosome read-through past the stall region relative to the wild-type strain (Figure S5B). Additionally, we noted from the disome profiling that strain as revealed by an autocorrelation analysis (Figures 5C, S5D). The same diminished periodicity is observed in monosomes in the hel2 $\Delta$ strain, whereas the ribosome periodicity is generally maintained in the syh1 1 strain (Figure S5C).

In contrast to the minCGA reporter, the minNONOPT reporter contains non-optimal codons 303 distributed throughout the ORF; accordingly, monosome footprints are relatively evenly distributed 304 across the ORF (Figure 5A, right panel). Disome footprints are distributed across the ORF as well, suggestive of stochastic short-lived collisions where the ribosome density does not accumulate. To allow 

the start and stop codons that can be sensitive to library preparation and variable within ribosome profiling data sets (O'Connor, Andreev, and Baranov 2016). Importantly, we normalized these data from the reporter ORF to global ORF 21-mer/28-mer ratios within each dataset to account for differing RNase digestion efficiencies in the library preparations. Our data reveal an increased 21-mer/28-mer ratio for the minNONOPT relative to the minOPT reporter; as 21-mer RPFs report on empty $A$ sites on the elongating ribosomes, these data are consistent with an enrichment of ribosomes collectively undergoing

314 slow decoding of tRNAs during elongation on non-optimal codons (Figure 5D). These data suggest that a 315 key signal for the recruitment of the COMD machinery (including Not5) is the accumulation of slowly decoding ribosomes. of the distribution of multiply aligned monosome footprint reads within the CGA repeat region of the minCGA reporter revealed that the $21 \mathrm{mer} / 28 \mathrm{mer}$ read ratio is greatly enriched compared to the ratio on

322 all genes (Figure 5E). We suggest that this preponderance of slow (21mer RPFs) elongating monosomes in 323 the CGA repeat region might explain the partial sensitivity of the NGD reporter to Not5-mediated 324 destabilization. Since Not5 recognizes ribosomes with open A and E sites, it may be able to bind those 325 ribosomes that continue translating within the $\mathrm{CGA}_{12}$ region to elicit decay of the reporter. 
accumulation of terminally stalled, collided ribosomes, while Not5 responds to slowly decoding ribosomes with open $A$ and $E$ sites.

\section{2}

\section{Discussion}

In this study, we use carefully designed reporter mRNAs to study translation-coupled mRNA decay pathways in S. cerevisiae. Using R-SGA screening with a reporter mRNA containing iterated CGA codons, we identified and validated a set of genes that contribute to no-go decay (NGD). Subsequent analysis allowed us to compare the mechanisms of the pathways that regulate decay of mRNAs with either highly problematic (NGD) or slowly decoded sequences (COMD). We find that NGD is driven primarily by the actions of the GIGYF1/2-homologous protein Syh1; in contrast, Syh1 has no discernible impact on the stability of non-optimal mRNA sequences. We show that the previously defined COMD factor Not5 contributes modestly to decay of the NGD reporter and very strongly to decay of non-optimally coded mRNAs. Finally, we connect these distinct molecular decay profiles with ribosome states using ribosome profiling, showing that colliding ribosomes (disomes) are the key trigger for Syh1/Hel2 function while slow ribosomes (monosomes with empty A and E sites) are the key trigger for Not5 function.

Our assays using the NGD reporters reveal the interplay between Hel2, its dependent NGD factors, and Syh1 in responding to ribosome collisions. First, we found that deletion of SYH1 led to modest stabilization of NGD reporter mRNAs and deletion of CUE2 and SYH1 together led to very potent stabilization (Figure 2C). In light of previous work, we interpret the mRNA stability data as follows: 1) in the wild-type strain, the NGD reporters are stabilized because colliding ribosomes lead to the recruitment of Syh1 and elicit mRNA destabilization through Xrn1 while Hel2-mediated ubiquitination triggers ribosomal clearance by SIh1; 2) in the syh1 strain, NGD reporter levels are somewhat rescued because recruitment of Xrn1 is impaired, but Cue2-mediated endonucleolytic decay plays a larger role as SIh1 becomes overwhelmed; and 3) in the hel2 $\Delta$ syh $1 \Delta$ and cue $2 \Delta$ syh $1 \Delta$ strains, NGD reporters are strongly 
stabilized because both exonucleolytic NGD through Xrn1 and endonucleolytic NGD through Cue2 are inactivated. This model gives new context to the pathways that respond to elongation stalls; it emphasizes the essentiality of Syh1 for Xrn1-mediated NGD and supports our previously discovered mechanism in which the activity of Hel2 primarily triggers RQT-mediated ribosome rescue through Slh1, resorting to Cue2-dependent endonucleolytic cleavage only when other mechanisms to resolve collisions are overwhelmed. The synergistic activities of Syh1-assisted, Xrn1-mediated decay and Hel2-assisted, Cue2mediated decay form the basis of a robust cellular system for targeting problematic mRNAs for destruction (Figure 6). in the WT strain, and this pattern was substantially disrupted in the hel2 $\Delta$ strain. Furthermore, we observe 364 a larger proportion of monosome footprints downstream of the CGA region in the hel2 $\Delta$ strains. Given the role of Hel2 in recognizing collided ribosome and promoting clearance by RQT, we interpret these data to mean that the activity of Hel2 on collided ribosomes stabilizes the structure and triggers clearance by Slh1

367 (Meydan and Guydosh 2020), thus preventing ribosome build-up in the downstream region. This stands 368 in contrast to the maintenance of wild-type-like periodicity when SYH1 is knocked out (Figure S5C). The next set of questions focused on how Syh1 is recruited to problematic mRNAs and how it 370 triggers translational repression or mRNA decay. Recent work in mammalian cells investigating the 371 mechanism of recruitment of GIGYF2 to NGD-targeted mRNAs yielded two competing models: one in 372 which ZNF598 (a mammalian HEL2 homolog) acts to recruit GIGYF2 (Hickey et al. 2020) and one in which 373 EDF1 acts to recruit GIGYF2 (Sinha et al. 2020; Juszkiewicz et al. 2020). We tested both models. First, we 374 observed strong stabilization of the CGA reporter mRNA levels when SYH1 is deleted in a hel2A

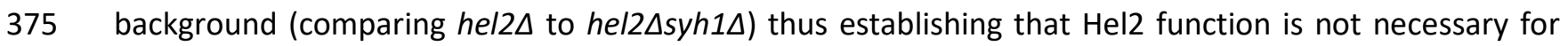
Syh1 function in yeast. Second, we observed the same strong repression of CGA reporter mRNA levels in 


\section{NGD}

hel $2 \Delta \operatorname{syh} 1 \Delta$

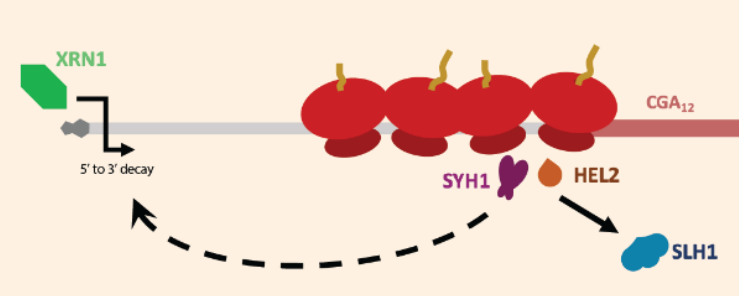

$\operatorname{syh} 1 \Delta$
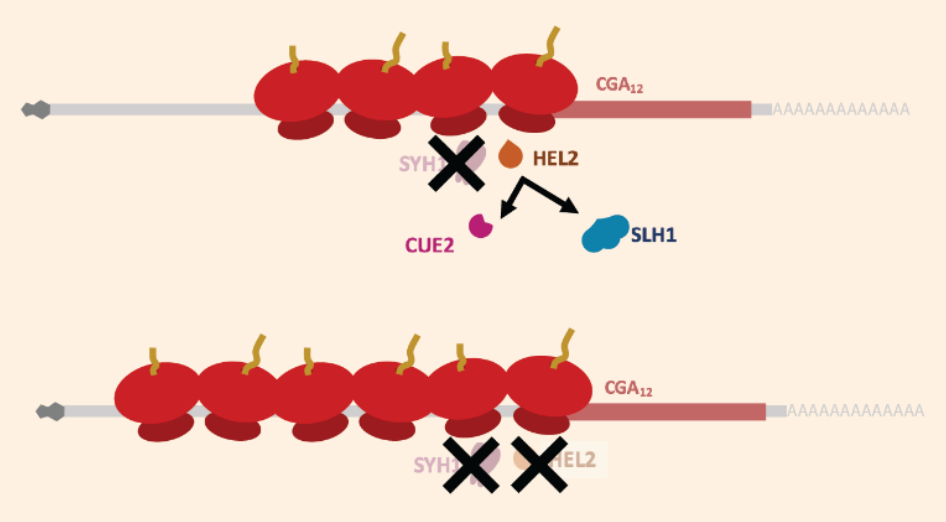

COMD

WT

$\operatorname{not} 5 \Delta$

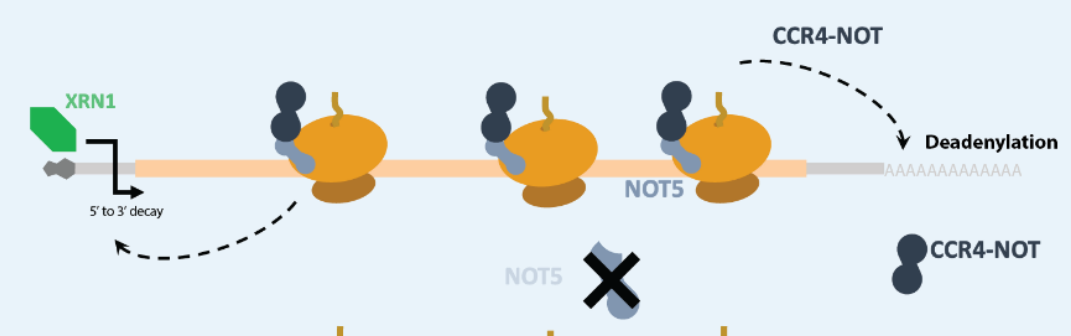

Figure 6: A model for NGD and COMD

In NGD, Syh1 responds to collided ribosomes, connecting severe translational blocks to mRNA decay through decapping and 5' to 3' decay by Xrn1. Loss of Syh1 results in activation of Hel2-dependent endonucleolytic NGD by Cue2. Loss of Syh1 and Hel2 causes increased reporter accumulation by blocking both exonucleolytic and endonucleolytic RNA decay pathways.

In COMD, Not5 senses slow ribosomes on non-optimal codons and recruits the Ccr4-Not complex, causing deadenylation, decapping, and 5' to $3^{\prime}$ decay. Loss of Not5 stabilizes non-optimal mRNAs. 
a wild-type strain and a knockout of the yeast EDF1 homolog MBF1 (a result that differs from the rescuing effect of SYH1 knockout), suggesting that Syh1 function is not dependent on Mbf1. Although Mbf1 binds collided disomes in yeast (Sinha et al. 2020; Pochopien et al. 2021), these data suggest that Mbf1 is not necessary for NGD in yeast. One possibility raised by biolD mass spectrometry (Opitz et al. 2017) is that Syh1 interacts directly with Asc1, a ribosomal protein known to be important for NGD in yeast (Kuroha et

(Figure 1A). It is possible that Syh1 detects collisions by direct association with collided disomes. translational repression. We note that the strong mRNA decay phenotype associated with Syh1 function 
recognized by Not5. Conversely, the possibility remained open that highly non-optimal reporter mRNAs cause ribosome collisions that activate NGD in addition to COMD.

We observe that the loss of NOT5 modestly stabilizes the minCGA reporter, suggesting that the non-optimal decay machinery can respond to slow ribosomes even at sites undergoing NGD. For this reporter, accumulated ribosome footprints from within the CGA-repeat region are short (21-mers), reflect ribosomes with unoccupied A sites as they struggle to decode this problematic sequence (D'Orazio et al. 2019). 21-mer RPFs are also enriched in the minNONOPT reporter. These short footprint reads on both reporters represent very slow ribosomes with open A and E sites that are recognized by Not5, leading to recruitment of the Ccr4-Not complex. This observation rationalizes the modest stabilization of the minCGA reporter and the strong stabilization of the minNONOPT reporter in the not5 $\Delta$ strain. In contrast, we saw no half-life increase of the minNONOPT reporter in the syh1 $1 \Delta$, hel2 $\Delta$, or hel2 $\Delta$ syh1 $1 \Delta$ strains, arguing that these abundant non-optimal codons do not induce ribosomal collisions that trigger NGD, consistent with

413 a relatively even distribution and low abundance of disome peaks in the ribosome profiling data. These data provide strong evidence that non-optimal sequences are recognized distinctly from NGD promoting 415 sequences. Our study provides strong evidence for generally non-overlapping targets and mechanisms of

417 NGD and COMD. While the NGD machinery, under the control of Syh1 and Hel2, responds to specific 418 defects in elongation due to stalled and collided ribosomes, the COMD machinery, under the control of 419 Not5, surveys the pool of translating ribosomes for mRNAs on which there is overall inefficient translation.

420 We speculate that the COMD pathway is a general one that regulates overall mRNA stability, independent

421 of ribosome dysfunction, while the NGD pathway evolved to deal with more acute environmental 422 disturbances such as UV or oxidative damage (Yan et al. 2019; C. C.-C. Wu et al. 2020). Future studies will 423 better characterize the molecular mechanisms of these pathways and will provide new foundations for 424 an understanding of the homeostasis of cellular translation and mRNA decay. 
bioRxiv preprint doi: https://doi.org/10.1101/2021.12.01.470814; this version posted December 1, 2021. The copyright holder for this preprint (which was not certified by peer review) is the author/funder, who has granted bioRxiv a license to display the preprint in perpetuity. It is made available under aCC-BY-NC-ND 4.0 International license.

A)
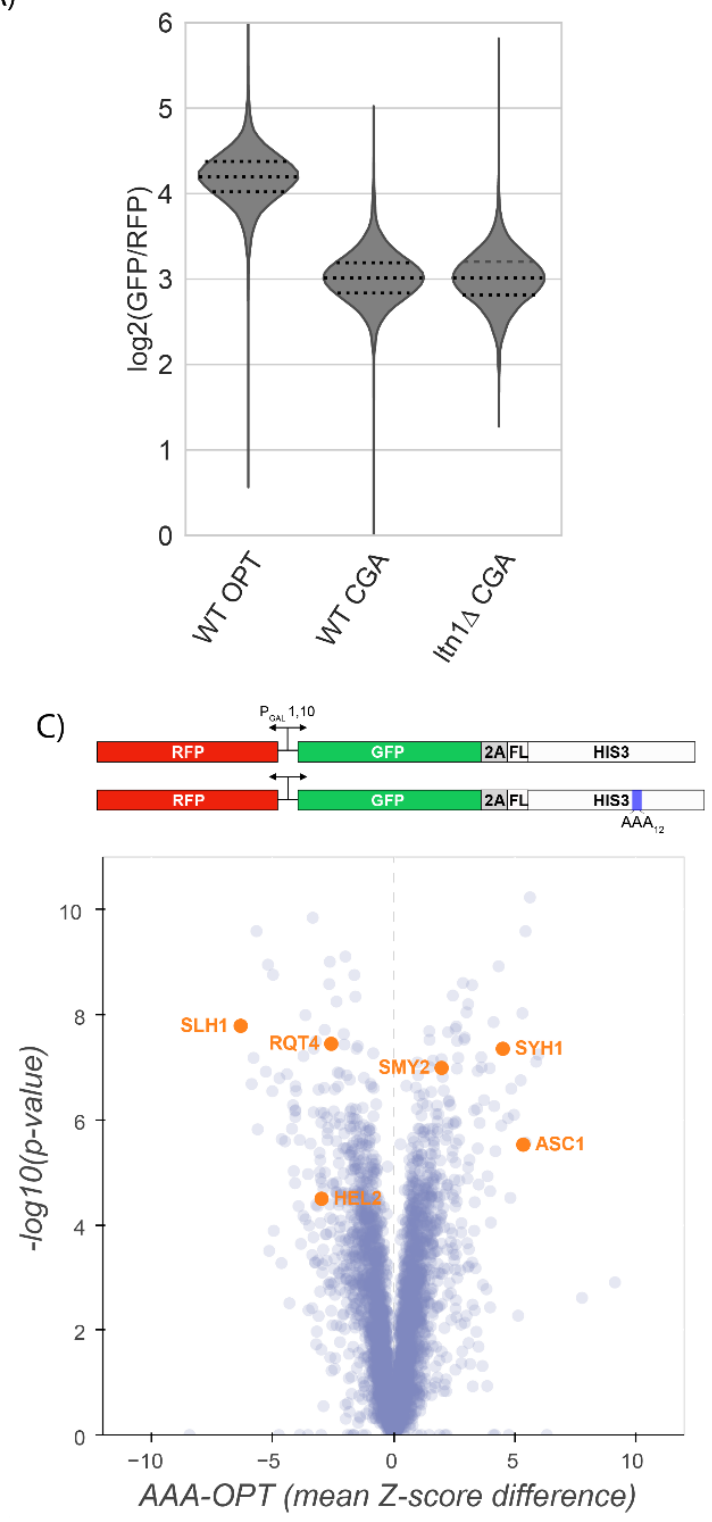

E)

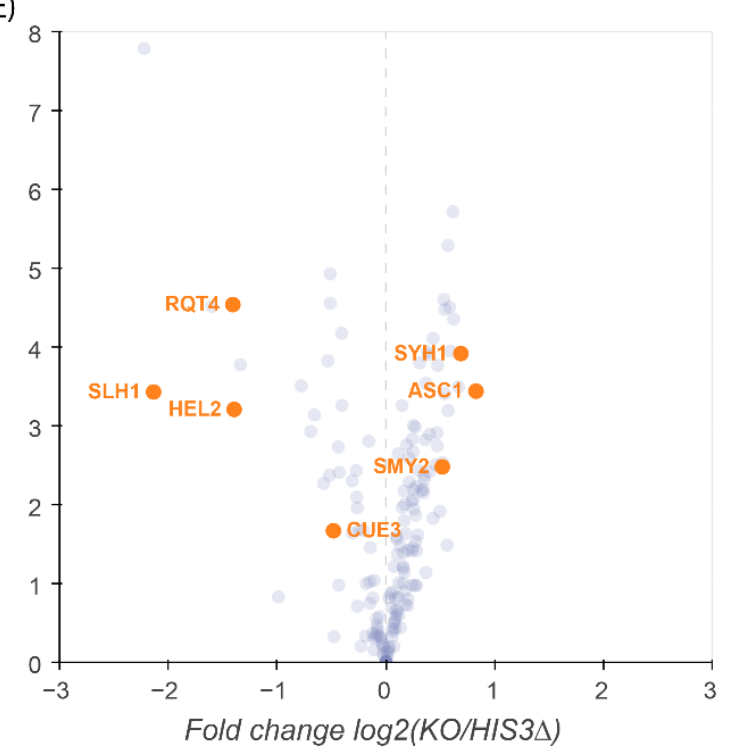

B)

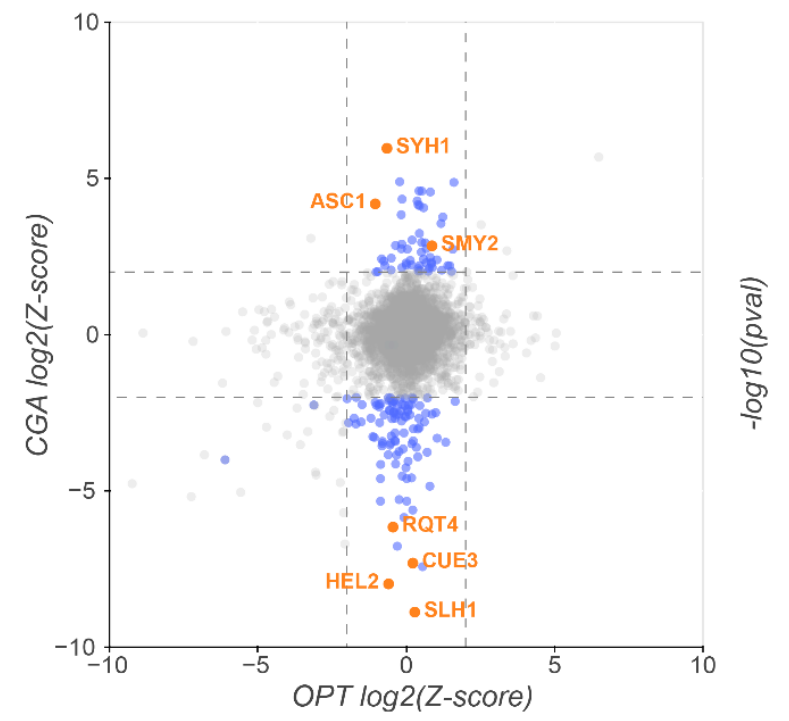

D)

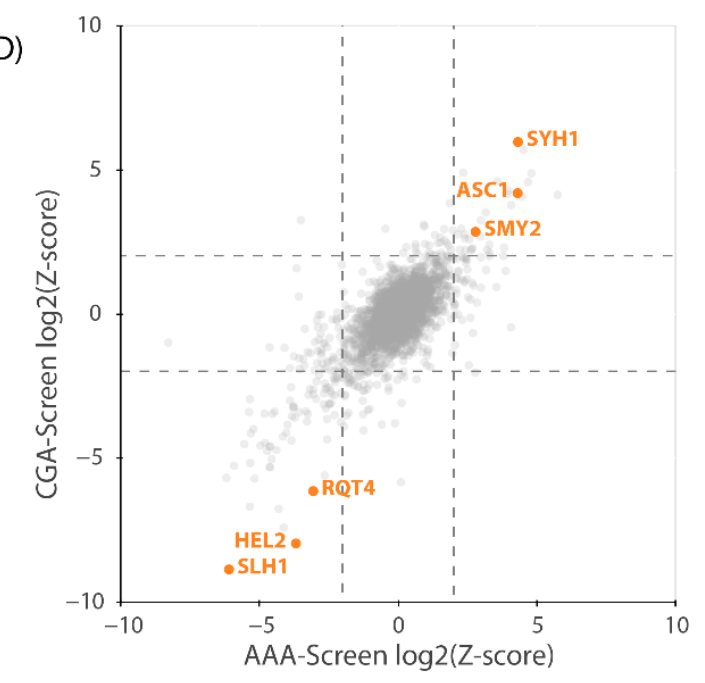

F)
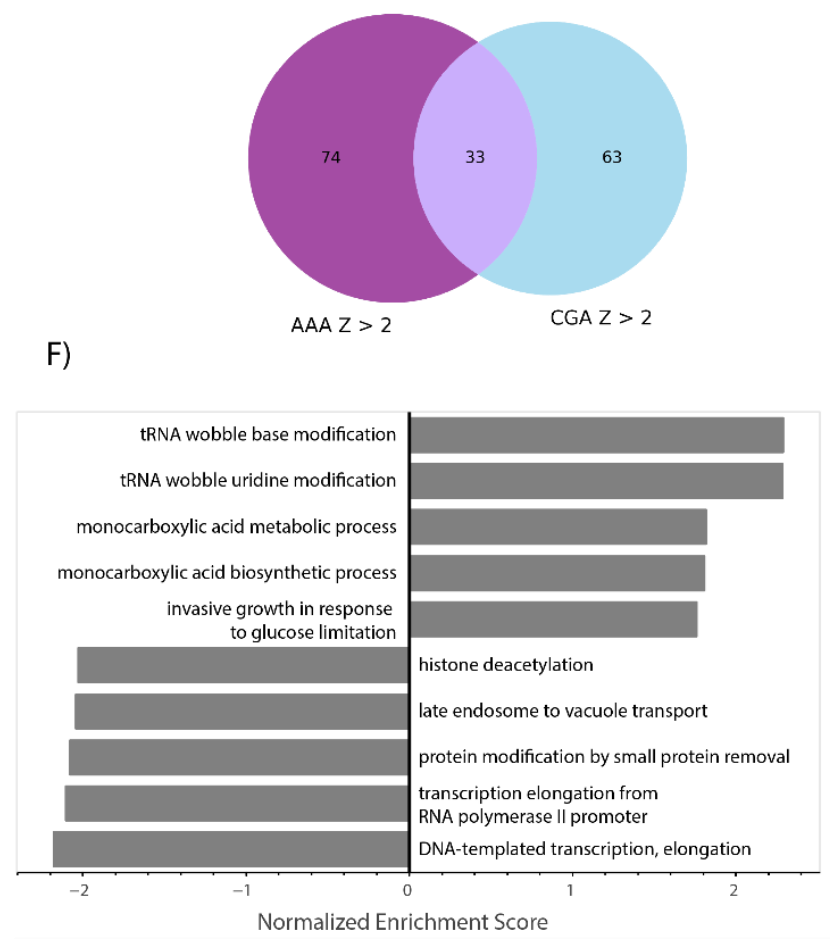
bioRxiv preprint doi: https://doi.org/10.1101/2021.12.01.470814; this version posted December 1, 2021. The copyright holder for this preprint

(which was not certified by peer review) is the author/funder, who has granted bioRxiv a license to display the preprint in perpetuity. It is made available under aCC-BY-NC-ND 4.0 International license.

\section{Supplemental Figure 1: Analysis of R-SGA screens and reporters}

A) Flow cytometry analysis of OPT and CGA GFP protein fluorescence reporter levels normalized to RFP fluorescence in WT and Itn1 $\Delta$ strains. Distributions represent log2(GFP/RFP) ratios of individual cells. Several known NGD factors are highlighted.

B) Normalized Z-scores based on data from Figure 1B plotted as per-plate z-scores for each gene in the OPT and CGA screens. Genes colored blue (-2 > CGA Z-score $>2$ and -2 < OPT Z-score < 2) were selected for follow-up validation.

C) Gene diagrams comparing the OPT and AAA reporters. Similar to the CGA reporter, a stretch of twelve AAA codons were inserted into the HIS3 ORF. Screening data comparing the OPT and AAA screens are plotted as in Figure 1B. Several known NGD factors are highlighted.

D) Normalized Z-scores based on data from Figure S1C from the AAA and CGA screens plotted against each other as in B to emphasize similarity between data from these screens. A Venn diagram shows the overlap between the genes with a Z-score greater than 2 in the CGA and AAA screens.

E) Volcano plot of flow cytometry screen validation with reporters freshly reinserted into knockout collection strains. Fold changes were calculated relative to WT his $3 \Delta$ knockout lines and p-values were obtained from a Fisher's t-test. Several genes are highlighted.

F) Ranked gene-set enrichment analysis for GO terms was performed. Top enriched GO terms from the GO Biological Process annotations were plotted. Positive enrichment scores indicate enrichment among CGA screen hits with positive Z-scores (i.e. knockouts causing increased CGA reporter levels) and vice versa for negative enrichment scores. 
bioRxiv preprint doi: https://doi.org/10.1101/2021.12.01.470814; this version posted December 1,2021 . The copyright holder for this preprint (which was not certified by peer review) is the author/funder, who has granted bioRxiv a license to display the preprint in perpetuity. It is made available under aCC-BY-NC-ND 4.0 International license.

A)

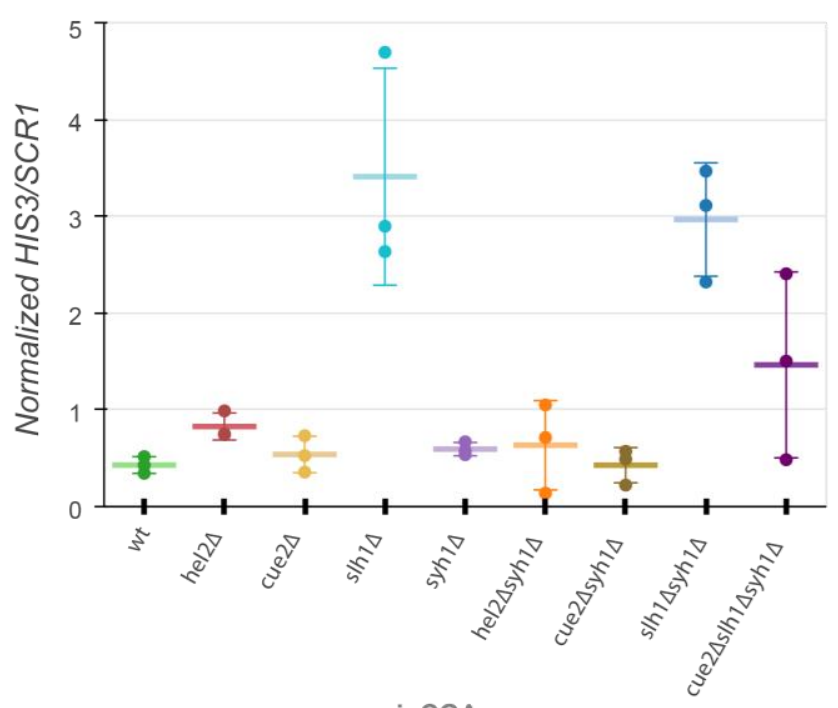

$\operatorname{minCGA}$

C)

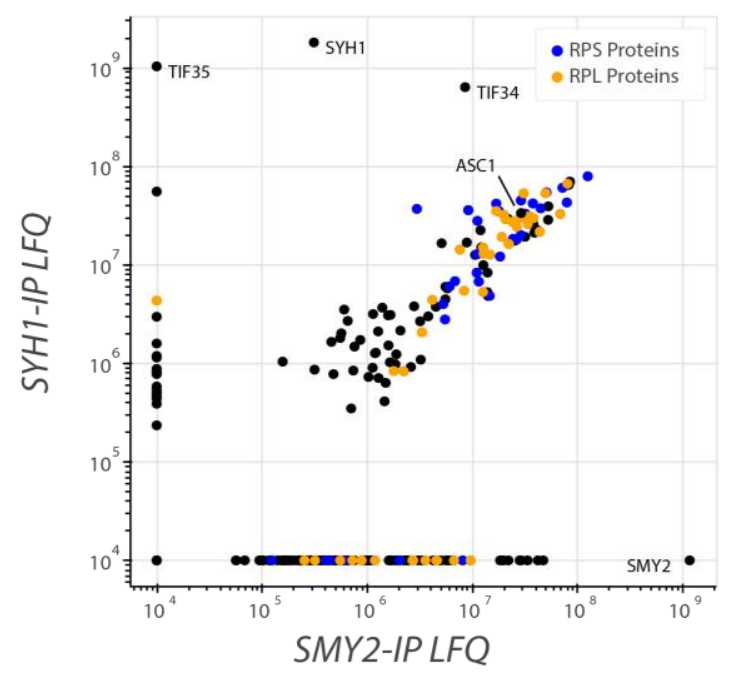

E)

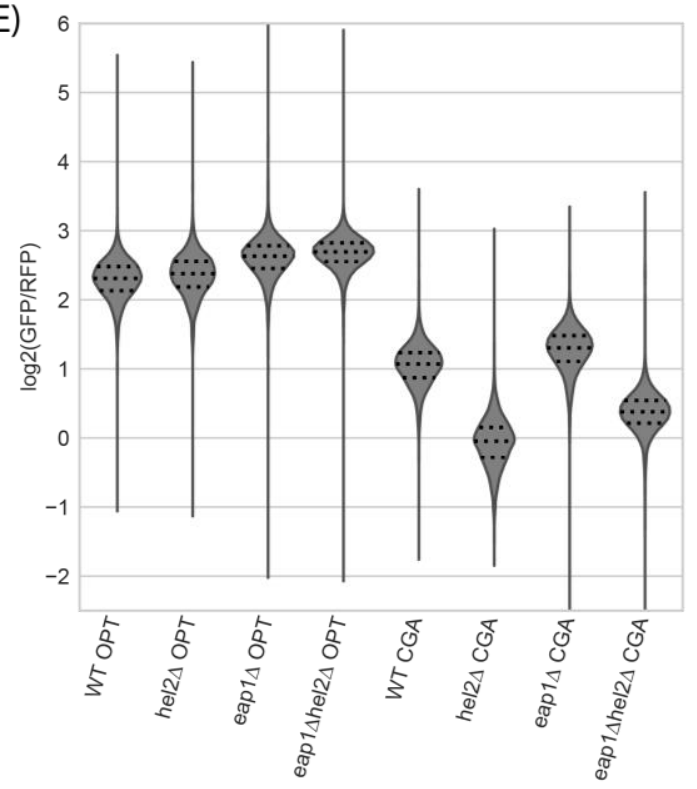

B)

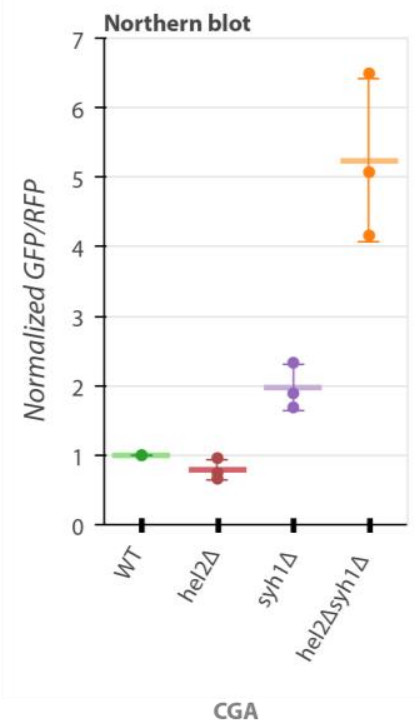

D)

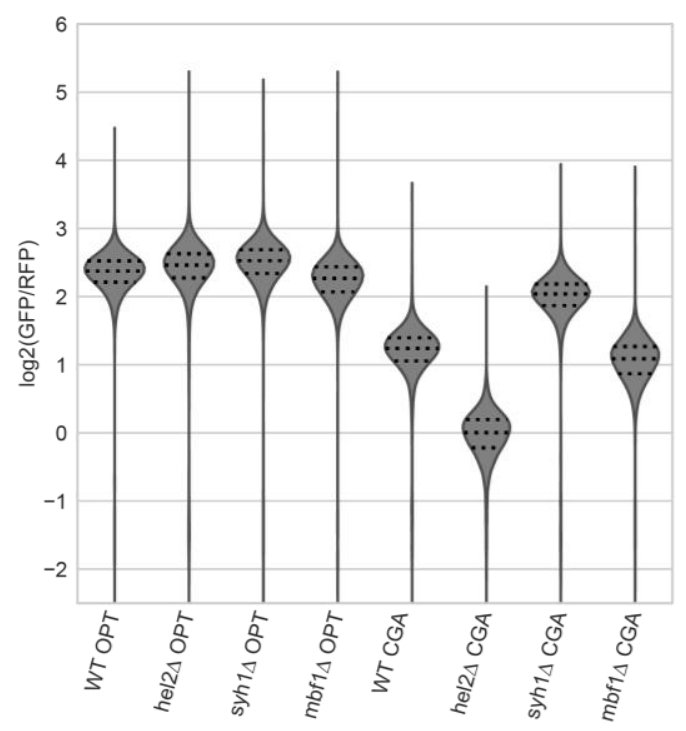

F)

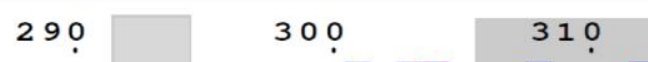

GIGYF1 GLPEWCLDDEDEEMTFDASGAF

GIGYF2 SLPEWCLEDAEEEMGTFDSSGAF

Syh1 $\ldots \ldots \ldots$ MDSIGVQRVP S PF

Smy2 $\ldots \ldots \ldots$ IDSIGIQRAAS P E 
bioRxiv preprint doi: https://doi.org/10.1101/2021.12.01.470814; this version posted December 1, 2021. The copyright holder for this preprint

(which was not certified by peer review) is the author/funder, who has granted bioRxiv a license to display the preprint in perpetuity. It is made available under aCC-BY-NC-ND 4.0 International license.

\section{Supplemental Figure 2: Analysis of factors affecting NGD}

A) Quantifications of the 3' fragment of the minCGA reporter measured by autoradiographic northern blot as in Figure 2C. Means from three biological replicates are plotted. Data was normalized within each replicate set to the full length WT CGA band as in Figure 2C. Error bars are standard deviation.

B) Quantifications of the full length GFP-containing CGA reporter measured by autoradiographic northern blot as in Figure 1D.

C) Affinity purification-mass spectrometry of Syh1-TAP and Smy2-TAP. LFQ values were calculated using MaxQuant software. Ribosomal (RPS and RPL) proteins are colored orange and blue.

D) Flow cytometry analysis in several strains including embf1 $\triangle$ of OPT and CGA GFP protein fluorescence reporter levels normalized to RFP fluorescence. Distributions represent $\log _{2}(\mathrm{GFP} / \mathrm{RFP})$ ratios of individual cells.

E) Flow cytometry analysis in several strains including eap1D of OPT and CGA GFP protein fluorescence reporter levels normalized to RFP fluorescence. Distributions represent $\log _{2}$ (GFP/RFP) ratios of individual cells.

F) Protein multiple sequence alignment produced by structure-aware alignment with T-Coffee Expresso (Notredame, Higgins, and Heringa 2000). Output diagram was generated by ESPript 3.0 (Robert and Gouet 2014). The GIGYF1/2 conserved binding motif regions from Weber et al. 2020 are indicated by gray shading. 
bioRxiv preprint doi: https://doi.org/10.1101/2021.12.01.470814; this version posted December 1, 2021. The copyright holder for this preprint (which was not certified by peer review) is the author/funder, who has granted bioRxiv a license to display the preprint in perpetuity. It is made available under aCC-BY-NC-ND 4.0 International license.

A)

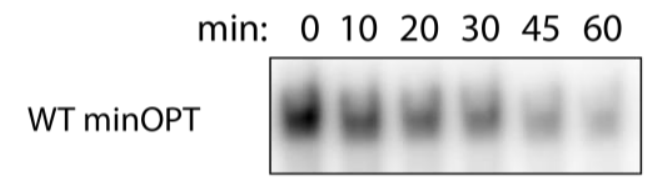

min: 01020304560 $\operatorname{syh} \Delta \operatorname{minOPT}$

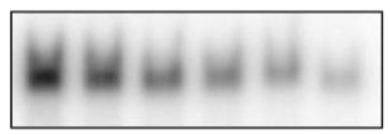

min: $0 \begin{array}{lllll}10 & 20 & 30 & 45 & 60\end{array}$
WT minCGA

min: $\begin{array}{llllll}0 & 2 & 5 & 10 & 20 & 60\end{array}$

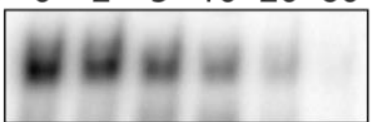

min: $0 \quad 10 \quad 2030 \quad 45 \quad 60$

$\operatorname{syh} 1 \Delta \operatorname{minCGA}$

min: $0 \quad 102030 \quad 4560$

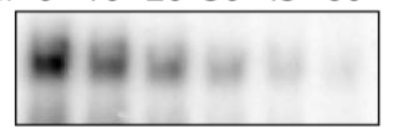

hel2 $2 \Delta$ syh1 $\Delta \operatorname{minOPT}$

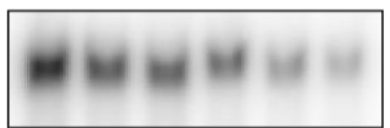

hel2 2 syh1 $\Delta$ minCGA

B)
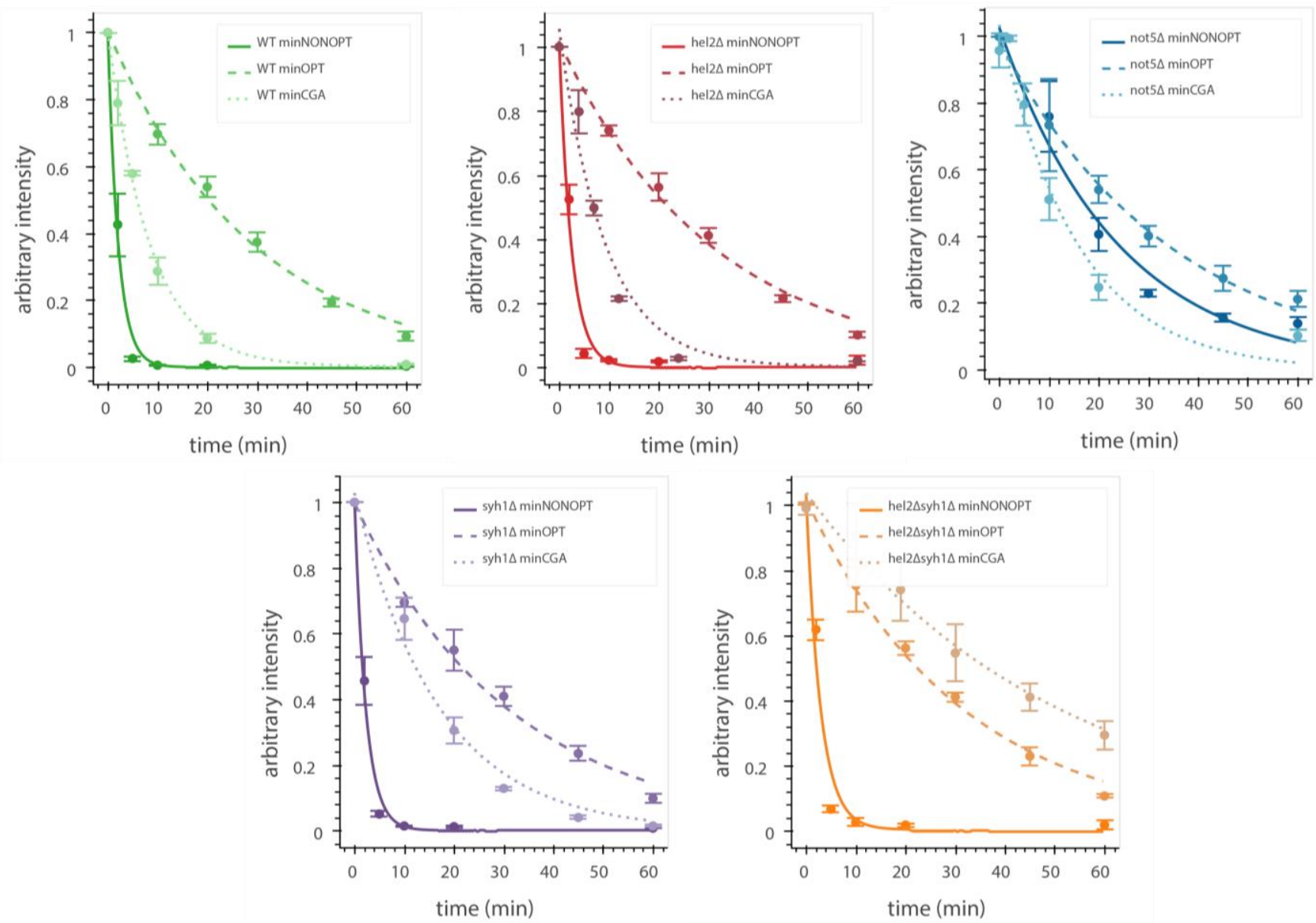
bioRxiv preprint doi: https://doi.org/10.1101/2021.12.01.470814; this version posted December 1, 2021. The copyright holder for this preprint (which was not certified by peer review) is the author/funder, who has granted bioRxiv a license to display the preprint in perpetuity. It is made available under aCC-BY-NC-ND 4.0 International license.

\section{Supplemental Figure 4: Galactose shutoff mRNA decay of reporters in knockout backgrounds}

A) Northern blot autoradiography images for reporter mRNAs in galactose shutoff assays in the indicated genetic backgrounds. Representative images are shown.

B) Decay curves for reporters from northern blots are plotted for different backgrounds as in Figure 4B. Reporter mRNA levels were normalized to endogenous SCR1 levels. Reporter mRNA decay curves measured by northern blot analysis after GAL promoter shutoff in different genetic backgrounds. A single exponential decay was fit to means of three or four replicates at each timepoint. 
bioRxiv preprint doi: https://doi.org/10.1101/2021.12.01.470814; this version posted December 1, 2021. The copyright holder for this preprint (which was not certified by peer review) is the author/funder, who has granted bioRxiv a license to display the preprint in perpetuity. It is made available under aCC-BY-NC-ND 4.0 International license.

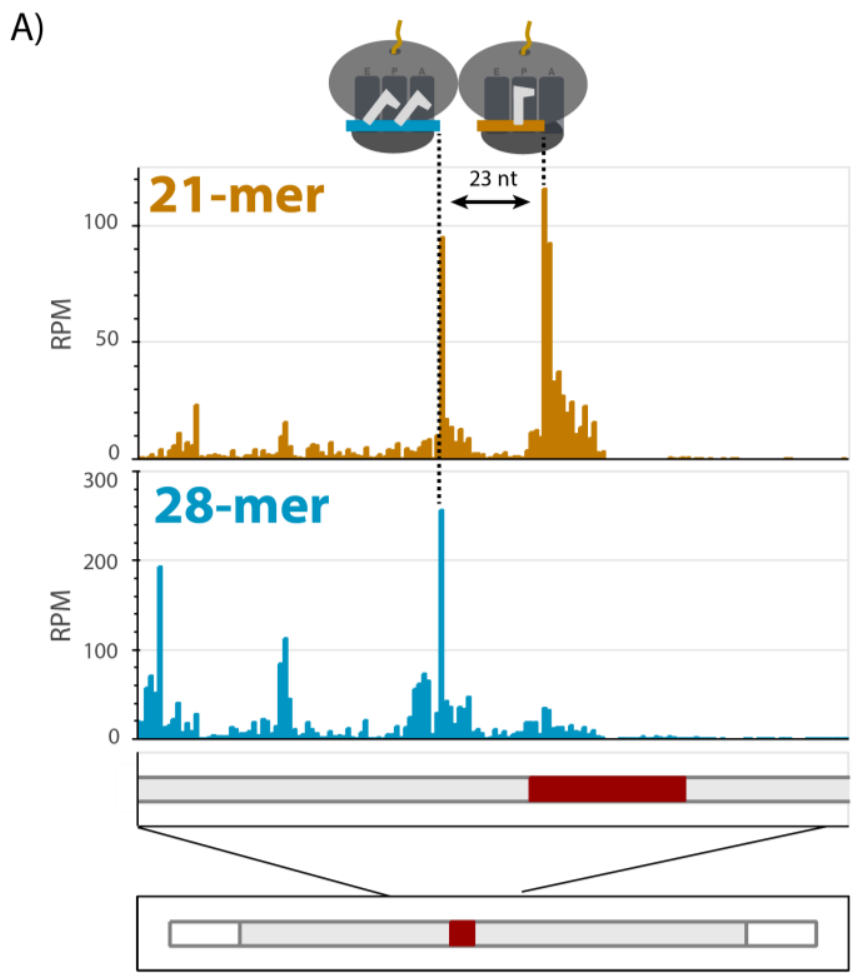

B)

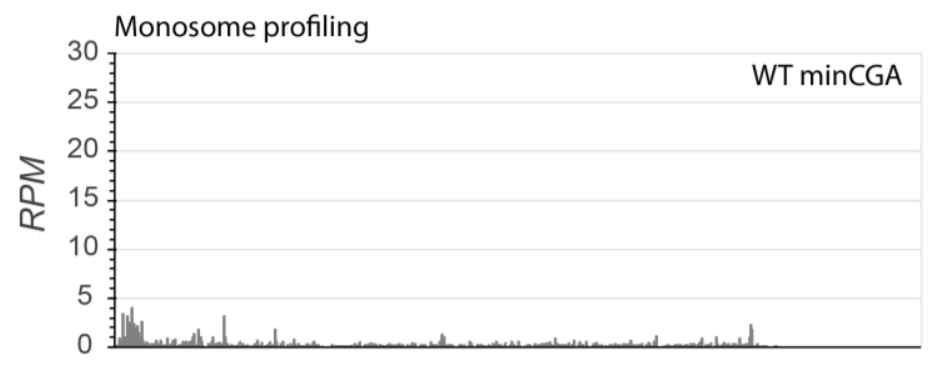

C)
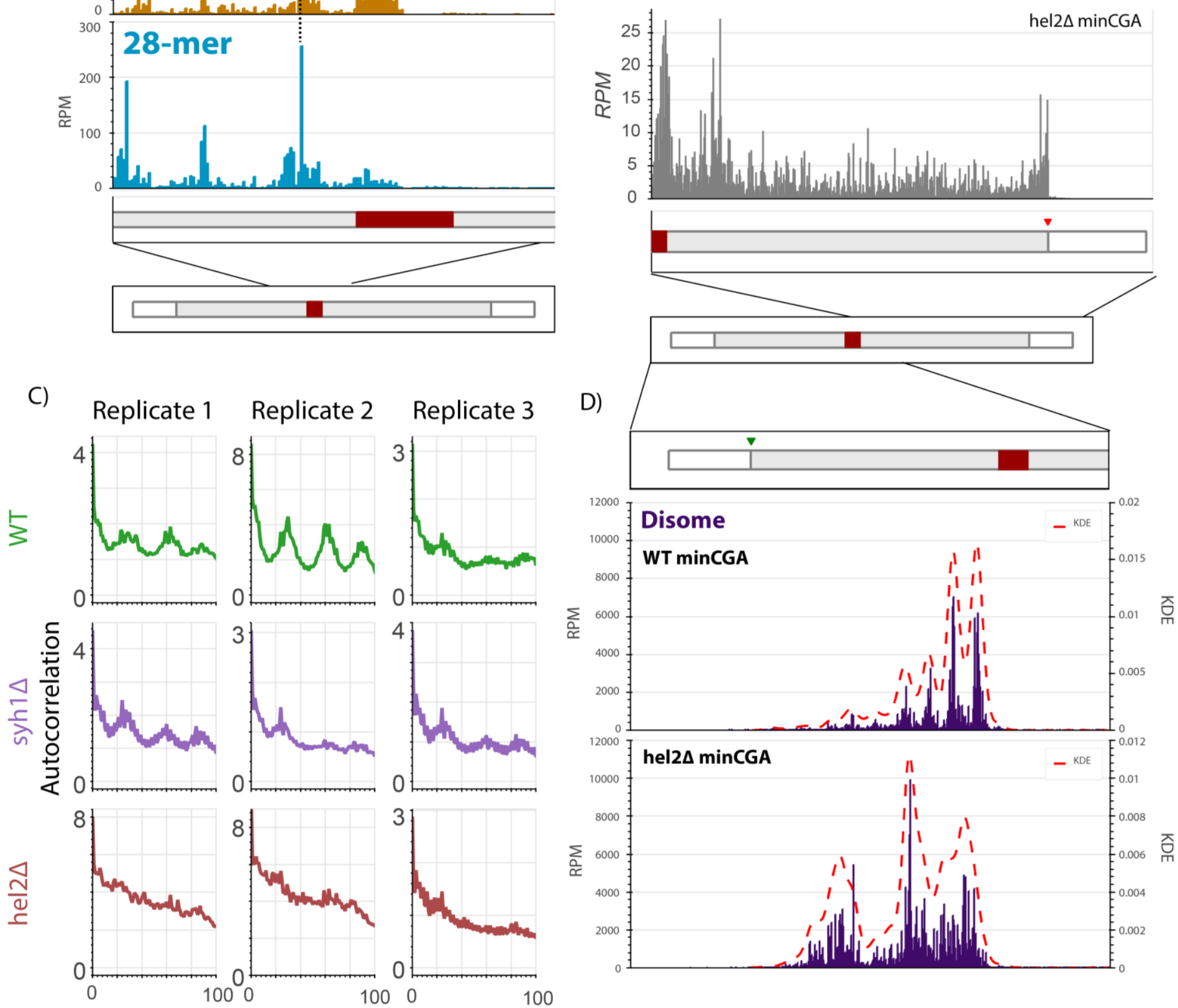

Displacement 
bioRxiv preprint doi: https://doi.org/10.1101/2021.12.01.470814; this version posted December 1, 2021. The copyright holder for this preprint (which was not certified by peer review) is the author/funder, who has granted bioRxiv a license to display the preprint in perpetuity. It is made available under aCC-BY-NC-ND 4.0 International license.

\section{Supplemental Figure 5: Ribosome profiling analysis}

A) RPMs for each position of the minCGA reporter replotted from Figure 5A enlarged to show the region around the CGA stall sequence. The leading 21-mer peak and the lagging 28-mer peak are approximately one ribosome length apart.

B) RPMs for each position of the reporter in monosome profiling data for the minCGA reporter replotted from Figure $5 \mathrm{~A}$ as a combination of all reads compared to the monosome profiling data from the hel $2 \Delta$ strain.

C) Autocorrelation of the footprint RPMs upstream of the stall plotted for three replicates in wild-type, syh1 $\Delta$, and $h e / 2 \Delta$ strains.

D) Read RPMs from disome footprint profiling are shown for each position of the minCGA reporter in the WT (top, replotted from Figure $5 \mathrm{~B}$ ) and hel2 $\Delta$ strains. A kernel density estimate (KDE) for each sample was superimposed to emphasize the periodicity of reads in the WT strain that is lost in the hel2 $\Delta$ strain. 


\section{Materials and Methods}

\section{Reporter cloning \& reporter strain generation}

Plasmids for OPT (pKD065), NONOPT (pKD064), and CGA (pKD080) reporters were cloned as

429 described in D’Orazio, et al. 2019. To generate stable, genomically integrated strains containing these

430 reporters, $0.5-2 \mu \mathrm{g}$ of plasmid was digested using Stul to produce an insertion cassette containing the RFP

431 and GFP reporters plus a MET17 gene for selection, all flanked by homology arms to the endogenous ADE2

432 locus. Strains were then transformed by lithium acetate transformation to replace the ADE2 gene as

433 described below, with the difference of being plated directly onto selective media after transformation

434 rather than a nonselective recovery plate. The minimal reporters minOPT and minNONOPT (plasmids

435 pJC867 and pJC857, respectively) were a generous gift from Jeff Coller and cloned as described in

436 Rhadakrishnan, et al. 2016. The CGA repeat stretch was introduced into the pJC867 plasmid by first

437 isolating the plasmid backbone via digestion with Pacl and Ascl. Two PCR fragments making up the HIS3

438 ORF were generated, one containing the first portion of the HIS3 ORF and a the CGA repeat region in a

439 primer overhang (primers AV_his3CGAupstr_fwd and AV_his3CGAupstr_rev, see table of oligos), the

440 other containing the downstream HIS3 sequence and stop codon (primers AV_his3CGAdwnstrm_fw and

441 AV_his3CGAdwnstrm_rv, see table of oligos). Both fragments were amplified off of pJC867 and inserted

442 into the linearized pJC867 backbone using NEB Gibson Assembly Master Mix resulting in pAV_minCGA

443 plasmid. Since these plasmids contained a URA3 selectable marker, reporters were introduced into

444 various backgrounds by transformation of 0.5-1 $\mu \mathrm{g}$ plasmid as described below and all subsequent culture

445 was performed in SC-URA media (plus additives appropriate to experiment) to retain the plasmid. 


\section{$446 \quad$ R-SGA screening}

447 Screening procedure

Screening was performed as described in D'Orazio et al, 2019. Briefly, OPT, CGA, NONOPT, and

449 AAA reporters were inserted into the Yeast Knockout Collection (Giaever et al. 2002) by mating and four

450 replicate colonies were grown for each strain. Incubation times were increased during this process by 50-

$45175 \%$ to account for decreased mating efficiency in our query strains. Cells were selected on appropriate

452 media, then plated on 2\% GAL/RAF media for expression and analyzed by imaging on a Typhoon FLA9500

453 imager.

454 Screen data analysis

Data was analyzed as previously described in D'Orazio et al. 2019 and D'Orazio et al. 2021. OPT

456 screen data used for normalization is the same data previously published in D'Orazio et al. 2021. Briefly,

457 median GFP and RFP values were extracted from colony images using specialized software (Saeed et al.

458 2003; Wagih et al. 2013) and outliers were excluded (border colonies and those $<1500$ or $>6000$ pixels).

459 Average GFP and RFP values from all colonies were then converted to $\log _{2}$ (GFP/RFP) ratios and LOESS

460 normalized on each plate. Z-scores were calculated on a per-plate basis. Z-scores for volcano plots were

461 calculated without prior LOESS normalization.

462 Screen validation

The 170 Yeast Knockout Collection strains with integrated reporters that showed the greatest 464 change in GFP/RFP for the CGA reporter (-2 > CGA Z-score > 2) and lowest change for the OPT reporter (-2

$465<$ OPT Z-score $<2$ ) relative to a his3 $\Delta$ control strain were selected and grown to saturation overnight in YP

$466+2 \%$ galactose $+2 \%$ raffinose media in deep-well 96 -well plates. Cultures were diluted in triplicate to 467 approximately $\mathrm{OD}_{600} 0.1$ in deep-well 96-well plates and grown to approximately $\mathrm{OD}_{600} 0.4-0.6$. A $10 \mu \mathrm{L}$ 
aliquot of culture was then added to $190 \mu \mathrm{L}$ PBS and flow cytometry was performed as described below in a Guava EasyCyte HT flow cytometer. P-values were calculated by Fishers T-test.

\section{Yeast strain generation, culture $\&$ harvesting}

\section{Knockout strain generation}

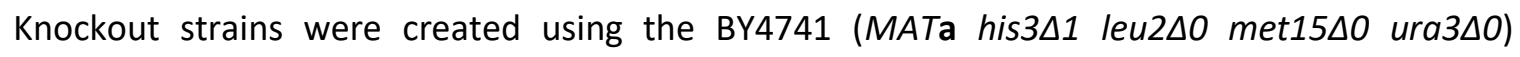

473 background as wild-type. DNA fragments containing 40-70 nt homology arms to the gene of interest were

474 amplified by PCR using MX cassette plasmids as template (McCusker 2017) and purified using a Zymo DNA

475 Clean \& Concentrator-5 kit. Yeast were then transformed using high-efficiency lithium acetate

476 transformation (Gietz and Schiestl 2007). Briefly, strains to be transformed were grown to saturation at

$47730^{\circ} \mathrm{C}$ overnight in an appropriate medium (typically YPD, YPAD, or SC-Ura), then diluted to $\mathrm{OD}_{600} 0.2$ in 5

$478 \mathrm{~mL}$ media. Meanwhile, the transformation mixture was prepared, consisting of 33\% PEG 3350, $100 \mathrm{mM}$

479 LiAc, $0.28 \mathrm{mg} / \mathrm{mL}$ boiled salmon sperm DNA, and 1-5 $\mu \mathrm{g}$ PCR product. When cultures reached $\mathrm{OD}_{600} 0.4-$

4800.6 , they were harvested by centrifugation $(3000 \mathrm{xg}, 5 \mathrm{~min})$ and resuspended in the transformation

481 mixture. Transformation mixtures were incubated at $42{ }^{\circ} \mathrm{C}$ (or $30^{\circ} \mathrm{C}$ for not $5 \Delta$ strains) on a thermomixer

482 for 40-60 minutes, then centrifuged briefly to collect a yeast pellet, discarding the supernatant. Finally,

483 yeast were resuspended in $200 \mu \mathrm{L}$ water (or media for not5 $\Delta$ strains), plated on an appropriate

484 nonselective agar medium and incubated at $30^{\circ} \mathrm{C}$. Transformants were then streaked to single colonies

485 on a fresh plate, and these colonies were tested for MX cassette insertion by PCR using Phire Plant Direct

486 PCR Master Mix. Confirmed strains were later maintained as patches on selective agar medium.

487 Growth conditions

Unless noted otherwise, yeast for steady-state reporter expression measurements (by flow 
grown to $\mathrm{OD}_{600} 0.4-0.65$ before being harvested according to the requirements of the particular assay to

492 be performed.

\section{Flow cytometry}

Cell lines to be analyzed with biological replicates were streaked to single colonies and three

495 individual colonies were selected for outgrowth and analysis. Cells were grown in liquid culture as

496 described above, then $500 \mu \mathrm{L}$ of cell culture was transferred to a microcentrifuge tube and pelleted by

497 centrifugation. Cells were washed once with PBS and then resuspended in $500 \mu \mathrm{L}$ PBS. Flow cytometry

498 was carried out using either a Guava EasyCyte or EasyCyte HT instrument, collecting $>5000$ events. Cellular

499 debris and dead cells were excluded on the basis of forward and side scatter, and geometric means of

500 per-cell GFP/RFP fluorescence distributions were used to calculate GFP/RFP for each replicate (Fig 1, 2A-

501 B, 3) or GFP/RFP ratios were calculated on an individual cell basis for plotting of distributions (Fig S2D-E).

502 For steady state measurements of OPT and CGA reporters in Figs $1 \mathrm{C}$ and 2A GFP/RFP ratios were further

503 normalized to the mean of WT OPT or WT CGA replicates, respectively, to place them on a similar scale to

504 northern blotting measurements.

\section{Galactose shutoff RNA half-life assay}

Biological replicates of individual cell lines were grown and diluted into $200 \mathrm{~mL}$ cultures in SC-URA

$507+2 \%$ Gal $+2 \%$ Raf media as described above. The $d h h 1 \Delta$ and not $5 \Delta$ strains were typically slow growing 508 and required longer incubations at $30{ }^{\circ} \mathrm{C}$ to reach saturation before dilution. When cultures reached $\mathrm{OD}_{600}$

509 0.4-0.6, they were split into four $50 \mathrm{~mL}$ conical tube and pelleted by centrifugation ( $3000 \times \mathrm{xg}, 5 \mathrm{~min}$ ). Cell

510 pellets were resuspended in $15 \mathrm{~mL}$ total prewarmed SC-URA media without added sugar to wash out

511 residual galactose and raffinose and pelleted again by centrifugation in a single $50 \mathrm{~mL}$ conical tube. Pellets

512 were resuspended in $10 \mathrm{~mL}$ prewarmed SC-URA without added sugar and transferred to a $125 \mathrm{~mL}$ beveled

513 flask in a shaking $30{ }^{\circ} \mathrm{C}$ incubator. Zero timepoints were taken by removing a $1 \mathrm{~mL}$ aliquot of culture, 
514 quickly transferring to a microcentrifuge tube and pelleting cells by a snap spin to $4000 \times \mathrm{xg}$. Supernatant

515 was decanted and tubes were dropped into liquid nitrogen. To initiate GAL promotor shutoff, $1 \mathrm{~mL} 40 \%$

516 glucose was added to the $9 \mathrm{~mL}$ remaining culture to a final concentration of $4 \%$ and a timer was started.

517 Subsequent timepoint samples were taken in a similar manner to the zero timepoint, with the time for

518 each sample recorded at the moment it was dropped into liquid nitrogen. All samples were stored at -80

$519{ }^{\circ} \mathrm{C}$. Downstream RNA extraction and northern blotting proceeded as described below.

\section{Steady state reporter cell harvesting for northern blot}

Cells were grown as described above in 10-15 mL. At $\mathrm{OD}_{600} 0.4-0.6$ cultures were pelleted at $4{ }^{\circ} \mathrm{C}$ was decanted. Tubes were dropped into liquid nitrogen and stored at $-80^{\circ} \mathrm{C}$ until RNA extraction.

\section{$525 \quad$ Northern blotting}

RNA extraction

RNA was extracted from frozen cell pellets by hot acid phenol/chloroform extraction. Aliquots of

$528325 \mu \mathrm{L}$ acid phenol, $\mathrm{pH} 4.5$ were heated to $65^{\circ} \mathrm{C}$ in microcentrifuge tubes on a thermomixer. Cell pellets

529 were retrieved from $-80^{\circ} \mathrm{C}$ storage and placed on dry ice. Working quickly, individual cell pellets were

531 until pellet was fully resuspended. One aliquot of preheated phenol was immediately added to the

532 resuspended pellet and sample was placed onto a thermomixer to minimize time between pellet

533 resuspension and cell lysis. This procedure was repeated for samples being processed in parallel, with

534 each sample shaking at the highest setting on the thermomixer for at least 15 minutes. Tubes were then

535 placed in a dry ice-ethanol bath for $\sim 30 \mathrm{~s}$ to help precipitate residual SDS and centrifuged at top speed for 
3 minutes. The top aqueous layer was placed in a new tube containing $300 \mu \mathrm{L}$ room temperature acid

537 phenol. Samples were vortexed several times for a total of 5 minutes, then centrifuged again at max speed

for $30 \mathrm{~s}$. The top aqueous layer was transferred to a tube containing $300 \mathrm{~mL}$ room temperature

chloroform, vortexed several times for a total of 5 minutes, and centrifuged at max speed for 3 minutes.

The aqueous phase was then transferred to a tube containing $30 \mu \mathrm{L} 3.5 \mathrm{M} \mathrm{NaOAc}$, pH 5.5. During each

547 water was added and samples were incubated at $37^{\circ} \mathrm{C}$ for 5 minutes on a thermomixer with gentle shaking samples were either used immediately for northern blotting or stored at $-80{ }^{\circ} \mathrm{C}$ for subsequent use. glass $500 \mathrm{~mL}$ beaker. This solution was heated in a microwave to boiling and agarose dissolution, mixed, then cooled to approximately $65^{\circ} \mathrm{C}$, placing an insulating material like paper towel beneath to promote even cooling of the solution. Particular care was taken not to allow the agarose to cool further than this

557 before formaldehyde addition, as pieces of unevenly cooled agarose can alter RNA mobility across the gel.

558 When initial cooling was complete, $8 \mathrm{~mL} 37 \%$ formaldehyde and $8 \mu \mathrm{L}$ ethidium bromide were added and 
560

561

562

563

564

565

566

567

568

569

570

571

572

573

574

575

576

577

578

579

580

581

582

583

formaldehyde gel running buffer (1x MOPS buffer, 1.67\% formaldehyde). Meanwhile, RNA samples were prepared by aliquoting an equal mass of total of RNA (typically $10 \mu \mathrm{g}$ ) into microcentrifuge tubes on ice containing an appropriate amount of 5x RNA loading buffer (bromophenol blue, 4 mM EDTA, 2.66\% formaldehyde, $20 \%$ glycerol, $30 \%$ formamide, $4 x$ MOPS buffer).

Gel running and transfer to membrane

RNA samples were boiled at $95{ }^{\circ} \mathrm{C}$ for 8 minutes, then cooled to room temp, spun briefly and loaded onto the gel. Gel was run at $100 \mathrm{~V}$ for $\sim 2.5 \mathrm{hrs}$. Gels were imaged on a Typhoon imager to assess RNA quality, then transferred to a Amersham Hybond N+ charged nitrocellulose membrane by a BioRad Model 785 Vacuum Blotter following the manufacturer's instructions for transferring RNA, with the alterations of prewetting the membrane with $10 x$ SSC only and maintaining vacuum between $10-15 \mathrm{inHg}$. Transfer proceeded for 2 hours. Following transfer, the membrane was carefully removed from the vacuum blotter and placed face up on paper towel for UV crosslinking in a Stratagene UV Stratalinker 2400 on the automatic setting $(120 \mathrm{~mJ})$ three times.

\section{Oligonucleotide probe radiolabeling and hybridization}

After crosslinking, the membrane was placed in a glass hybridization bottle with the RNA-side facing away from the glass. Approximately $15 \mathrm{~mL}$ Sigma Perfecthyb Plus Hybridization Buffer was added to the bottle and it was placed in a hybridization oven to prewarm for $30 \mathrm{~min}$ at $42{ }^{\circ} \mathrm{C}$. Meanwhile, the appropriate oligonucleotide probe was enzymatically radiolabeled with the final reaction concentrations $1 \mu \mathrm{M}$ oligonucleotide probe, 1x NEB T4 PNK buffer, 3-6 $\mu \mathrm{L}$ Perkin Elmer gamma- ${ }^{32} \mathrm{P}-\mathrm{ATP}, 25$ units NEB T4 PNK in a $50 \mu \mathrm{L}$ reaction volume. This reaction was incubated at $37^{\circ} \mathrm{C}$ for $1 \mathrm{hr}$, then the probe was purified using Cytiva Microspin G-50 columns according to the manufacturer's instructions. The entire volume of probe was then added directly to the prewarmed hybridization solution in the hybridization bottle. Membrane and radiolabeled probe were incubated at $42{ }^{\circ} \mathrm{C}$ with rotation overnight. The radioactive hybridization solution was discarded and the membrane was washed three times for 20 minutes each 
584 with $15 \mathrm{~mL}$ low-stringency wash buffer (0.1\% SDS, $2 \times \mathrm{SSC})$ at $30^{\circ} \mathrm{C}$. The membrane was placed between

585 transparency film or sheets of plastic wrap and secured into a phosphor storage screen cassette. A blanked

586 phosphor storage screen was exposed to the radioactive membrane long enough to produce adequate

587 exposure (typically overnight) and imaged as described below. To strip hybridized probe off of the

588 membrane, boiling high-stringency wash buffer (0.1\% SDS, $0.2 \times$ SSC) was poured on the membrane in a

589 hybridization bottle, incubated for 10 minutes at $80^{\circ} \mathrm{C}$, then discarded. The stripping procedure was

590 repeated for a total of two washes, then secondary probing was performed. For experiments with the

591 OPT, CGA, and NONOPT reporters, an oligonucleotide probe for GFP was used as the primary probe and

592 a probe for RFP as the secondary probe. For experiments with minOPT, minCGA, and minNONOPT

593 reporters, a probe for HIS3 was used as the primary probe and a probe for the endogenous yeast 7S RNA

594 SCR1 was used as the secondary probe.

595 Phosphor imaging, northern quantification, and half-life calculation

596 Phosphor storage screens were scanned with a typhoon imager and bands were quantified with

597 ImageQuant TL v8.1 software using rolling ball background subtraction. For each blot, the intensity of the

598 primary probe band was normalized to the intensity of the secondary probe band (GFP/RFP for OPT, CGA,

599 and NONOPT; HIS3/SCR1 for minOPT, minCGA, minNONOPT). For galactose shutoff experiments,

600 intensities and timepoints for three or four replicates were fit to a single-exponential decay by least-

601 squares fitting to estimate reporter RNA half-lives. For steady state reporter experiments,

602 reporter/control ratios in Figures 1D and 2B were further normalized to WT OPT and WT CGA within each

603 replicate set, respectively, to correct for any variation between individual blots. 


\section{Ribosome profiling}

605

606

607

608

609

610

611

612

613

614

615

616

617

618

619

620

621

622

623

624

625

626

627

Sample preparation

Ribosome profiling was carried out based on previously published protocols (McGlincy and Ingolia 2017; Guydosh and Green 2014; C. C.-C. Wu et al. 2019).

\section{Culture and ribosome RNA isolation}

Cultures were grown to saturation in appropriate media as described above and diluted to $\mathrm{OD}_{600}$

0.1 in $1 \mathrm{~L}$ culture. When cells reached $\mathrm{OD}_{600} 0.4-0.6$, cells were harvested by vacuum filtration and pellets were frozen in liquid nitrogen. A portion of each pellet was ground in a SPEX SamplePrep 6870 Freezer/Mill (8 cycles, 10 hz, 1 min run, 1 min cool) with $1 \mathrm{~mL}$ pre-frozen lysis buffer ( $20 \mathrm{mM}$ Tris pH 8, $140 \mathrm{mM} \mathrm{KCl,} 5$ $\mathrm{mM} \mathrm{MgCl}$, $1 \%$ Triton $\mathrm{X}-100,0.1 \mathrm{mg} / \mathrm{mL}$ cycloheximide, $0.1 \mathrm{mg} / \mathrm{mL}$ tigecycline) and thawed into $15 \mathrm{~mL}$ lysis buffer. Lysates were cleared by centrifugation $\left(5 \mathrm{~min}, 3000 \mathrm{xg}, 4{ }^{\circ} \mathrm{C}\right.$ ) and supernatants were loaded onto $3 \mathrm{~mL}$ sucrose cushion ( $20 \mathrm{mM}$ Tris $\mathrm{pH} 8,150 \mathrm{mM} \mathrm{KCl}, 5 \mathrm{mM} \mathrm{MgCl} 2,500 \mu \mathrm{M}$ DTT, $1 \mathrm{M}$ Sucrose) in a Ti70 ultracentrifuge rotor tube. Samples were centrifuged for 106 minutes at 60,000 RPM, $4{ }^{\circ} \mathrm{C}$ to pellet ribosomes. Supernatant was removed, and the ribosome pellet was rinsed once with lysis buffer excluding cycloheximide and tigecycline (drug-free lysis buffer). Pellet was resuspended by pipetting in $1 \mathrm{~mL}$ drugfree lysis buffer. RNA concentrations were measured by Qubit RNA High Sensitivity Assay Kit, $350 \mu \mathrm{g}$ of RNA was added to a microcentrifuge tube, and volume was increased to at least $400 \mu \mathrm{L}$ with drug-free lysis buffer. $5 \mu \mathrm{L}$ Ambion RNasel was added per $400 \mu \mathrm{L}$ of RNA solution, and samples were incubated at $25{ }^{\circ} \mathrm{C}$ in a thermomixer shaking at 500 RPM too digest free RNA. Samples were placed on ice and $10 \mu \mathrm{L}$ Superase $\bullet$ In RNase inhibitor was added and mixed to stop the RNase digestion. Sucrose gradients were prepared by a Biocomp Gradient Master (15-40\% sucrose gradient containing $20 \mathrm{mM}$ Tris pH 8, $150 \mathrm{mM}$ $\mathrm{KCl}, 5 \mathrm{mM} \mathrm{MgCl}$, $500 \mu \mathrm{M} \mathrm{DTT}$ ) in SW41 ultracentrifuge rotor tubes and RNase reactions were loaded in top of the gradients. Gradients were centrifuged at $40,000 \mathrm{RPM}$ for $2.5 \mathrm{hr}$ at $4{ }^{\circ} \mathrm{C}$. Gradients were 
a 15\% TBE-urea gel, taking care to leave empty lanes between samples to minimize cross-contamination.

Monosome libraries between 15 and $35 \mathrm{nt}$ (monosomes) or 40 and $70 \mathrm{nt}$ (disomes) were cut out of the

$647 \mathrm{NaOH}$ and incubating at $95^{\circ} \mathrm{C}, 5 \mathrm{~min}$. Samples were again purified with Zymo Oligo Clean \& Concentrator $648 \quad$ kit and eluted in $5 \mu \mathrm{L}$ nuclease free water. 
652 Oligos were annealed in a thermocycler, denaturing $90 \mathrm{~s}$ at $100{ }^{\circ} \mathrm{C}$, then dropping $0.1{ }^{\circ} \mathrm{C} / \mathrm{s}$ to $37{ }^{\circ} \mathrm{C}$ and

653 incubating 15 mins. MyOne Streptavidin C1 magnetic beads were prepared for RNA binding per the

654 manufacturers protocol and annealed oligo solutions were transferred to the beads. Solutions were

655 incubated for 15 mins at $37^{\circ} \mathrm{C}$, beads were pelleted and supernatants were transferred to new tubes.

656 Samples were cleaned up using Zymo Oligo Clean \& Concentrator kit and eluted in $6 \mu \mathrm{L}$ nuclease free

657 water.

658 Final sequencing library preparation

Loading dye was added to samples, and they were run on a $10 \%$ TBE-urea gel. With the aid of

gel slices as before with DNA extraction buffer (300 $\mu \mathrm{M} \mathrm{NaOAc}$ pH 5.5, 1 mM EDTA pH 8, 10 mM Tris pH8).

DNA was isopropanol precipitated, resuspended in $20 \mu \mathrm{L}$ circularization reaction mix (7.75 mM Tris pH 8,

1x Epicentre CircLigase buffer, $50 \mu \mathrm{M}$ ATP, $2.5 \mathrm{mM} \mathrm{MnCl}_{2}, 50$ units Circligase), incubated at $60{ }^{\circ} \mathrm{C}$ for $2 \mathrm{hr}$

and $80^{\circ} \mathrm{C}$ for $10 \mathrm{~min}$. Relative cDNA library abundances were tested by qPCR with BioRad iTaq Universal

665 SYBR Green Supermix to identify an appropriate number of PCR amplification cycles for each library. PCR

reactions were then performed for the determined number of cycles to introduce sequencing barcodes

667 and amplify libraries (1x Phusion HF buffer, $200 \mu \mathrm{M}$ dNTPs, $0.5 \mu \mathrm{M}$ oBZ287 universal forward PCR primer,

$6681 \mu \mathrm{M}$ reverse barcode PCR primer, 7.5\% v/v cDNA template, 1 unit Phusion polymerase). Samples were

669 mixed with loading dye and loaded on an 8\% TBE PAGE gel. Gel was run 60 min, $200 \mathrm{~V}$ and each library

670 was cut from the gel, frozen, and extracted from the gel slice as above using DNA gel extraction buffer.

671 Libraries were isopropanol precipitated, resuspended in $6 \mu \mathrm{L} 10 \mathrm{mM}$ Tris $\mathrm{pH}$ 8, and assessed for quality

672 and concentration using an Agilent BioAnalyzer 2100 High Sensitivity DNA assay. Libraries were pooled

673 and sequenced at the Johns Hopkins University Genetic Resources Core Facility on an Illumina NovaSeq

6746000 instrument. 


\section{Data processing}

Reads from raw FASTQ files were trimmed and aligned using a custom Python script to run software from the BBtools suite (https://jgi.doe.gov/data-and-tools/bbtools/) and the STAR aligner (https://github.com/alexdobin/STAR). Subsequent analyses were performed by custom Python scripts. Briefly, reads on start codons from all genes in monosome libraries were used to calculate distances from the $5^{\prime}$ end of a read to the ribosomal A site (17 nt for monosomes, $50 \mathrm{nt}$ for disomes). Reads per million mapped reads (RPMs) were calculated at each position of the reporter RNAs by dividing the number of $A$ site shifted $5^{\prime}$ ends at a given position by the total number of reads mapped to the genome (not including those that mapped to ncRNA). Reads with lengths 19 to 26 nt were considered part of the 21-mer population and reads with lengths 27 to $35 \mathrm{nt}$ were considered part of the 28 -mer population. Ratios of 21-mers/28-mers were calculated by dividing read numbers of 21-mers on the reporter (excluding the FLAG tag, five codons upstream of the top codon and the common binding region of the northern blotting probe) by read numbers of 28 -mers on the reporter and normalizing to the 21 -mer/28-mer ratio for all genes in a sample (Fig 5E). Ratios of 21-mers/28-mers in the CGA region of the minCGA reporter were calculated by re-aligning unaligned reads allowing multimapping (STAR option --outFilterMultimapNmax 999), then excluding any reads outside the CGA region and counting each unique read only once.

\section{Gene set enrichment analysis (GSEA)}

Using data from the CGA screen ranked by per-plate Z-score without LOESS normalization, ranked GSEA was performed using the GSEApy library for Python, querying the GO Biological Process annotation (“GSEApy," n.d.; Xie et al. 2021; Subramanian et al. 2005). The top five most enriched terms in each direction were selected for plotting. Full results are available in the supplemental files. 


\section{Affinity purification-mass spectrometry (AP-MS)}

TAP-tagged Syh1 and Smy2 strains were purchased from Dharmacon and grown as described

Hopkins University Mass Spectrometry and Proteomics Core facility and processed by facility personnel

searching against the UniProt yeast database and LFQ values for identified proteins were calculated without imputation, combining data from all fractions of each sample.

\section{Multiple Sequence Alignment}

Structure-aware multiple sequence alignment for human GIGYF1 (NCBI accession: 075420.2),

712 accession: NP_015220.1) was performed by T-Coffee Expresso (Notredame, Higgins, and Heringa 2000).

713 An additional alignment was performed by EMBL-EBI MUSCLE (https://www.ebi.ac.uk/Tools/msa/muscle)

714 to independently verify alignment results. T-Coffee Expresso alignment results were processed with

715 ESPript 3.0 (Robert and Gouet 2014) and output was included as Figure S2F. Regions corresponding to the

716 DDX6 binding motif identified in Weber et al. 2020 were shaded. 


\section{References}

Amberg, David C., Daniel J. Burke, and Jeffrey N. Strathern. 2006. "Tandem Affinity Protein (TAP) Purification from Yeast." CSH Protocols 2006 (1): db.prot4153.

Brandman, Onn, Jacob Stewart-Ornstein, Daisy Wong, Adam Larson, Christopher C. Williams, Gene-Wei $\mathrm{Li}$, Sharleen Zhou, et al. 2012. "A Ribosome-Bound Quality Control Complex Triggers Degradation of Nascent Peptides and Signals Translation Stress." Cell 151 (5): 1042-54.

Brown, Jeremy D., and Martin D. Ryan. 2010. "Ribosome 'Skipping': 'Stop-Carry On' or 'StopGo' Translation." Recoding: Expansion of Decoding Rules Enriches Gene Expression. https://doi.org/10.1007/978-0-387-89382-2_5.

Buschauer, Robert, Yoshitaka Matsuo, Takato Sugiyama, Ying-Hsin Chen, Najwa Alhusaini, Thomas Sweet, Ken Ikeuchi, et al. 2020. "The Ccr4-Not Complex Monitors the Translating Ribosome for Codon Optimality." Science (New York, N.Y.) 368 (6488): eaay6912.

Cosentino, G. P., T. Schmelzle, A. Haghighat, S. B. Helliwell, M. N. Hall, and N. Sonenberg. 2000. "Eap1p, a Novel Eukaryotic Translation Initiation Factor 4E-Associated Protein in Saccharomyces Cerevisiae." Molecular and Cellular Biology 20 (13): 4604-13.

Doma, Meenakshi K., and Roy Parker. 2006. "Endonucleolytic Cleavage of Eukaryotic MRNAs with Stalls in Translation Elongation." Nature 440 (7083): 561-64.

D’Orazio, Karole N., and Rachel Green. 2021. "Ribosome States Signal RNA Quality Control." Molecular Cell 81 (7): 1372-83.

D’Orazio, Karole N., Laura N. Lessen, Anthony J. Veltri, Zachary Neiman, Miguel Pacheco, Raphael LollKrippleber, Grant W. Brown, and Rachel Green. 2021. "Genetic Screens Identify Connections between Ribosome Recycling and Nonsense Mediated Decay." BioRxiv. https://doi.org/10.1101/2021.08.03.454884.

D’Orazio, Karole N., Colin Chih-Chien Wu, Niladri Sinha, Raphael Loll-Krippleber, Grant W. Brown, and Rachel Green. 2019. "The Endonuclease Cue2 Cleaves MRNAs at Stalled Ribosomes during No Go Decay." ELife 8 (June). https://doi.org/10.7554/eLife.49117.

Ergüden, Bengü. 2019. "Dhh1 Is a Member of the SESA Network." Yeast 36 (2): 99-105.

Fillingham, Jeffrey, Pinay Kainth, Jean-Philippe Lambert, Harm van Bakel, Kyle Tsui, Lourdes Peña-Castillo, Corey Nislow, et al. 2009. "Two-Color Cell Array Screen Reveals Interdependent Roles for Histone Chaperones and a Chromatin Boundary Regulator in Histone Gene Repression." Molecular Cell 35 (3): 340-51.

Gamble, Caitlin E., Christina E. Brule, Kimberly M. Dean, Stanley Fields, and Elizabeth J. Grayhack. 2016. "Adjacent Codons Act in Concert to Modulate Translation Efficiency in Yeast." Cell 166 (3): 67990.

Giaever, Guri, Angela M. Chu, Li Ni, Carla Connelly, Linda Riles, Steeve Véronneau, Sally Dow, et al. 2002. "Functional Profiling of the Saccharomyces Cerevisiae Genome." Nature 418 (6896): 387-91.

Gietz, R. Daniel, and Robert H. Schiestl. 2007. "High-Efficiency Yeast Transformation Using the LiAc/SS Carrier DNA/PEG Method." Nature Protocols 2 (1): 31-34.

Glover, Marissa L., A. Max Burroughs, Parissa C. Monem, Thea A. Egelhofer, Makena N. Pule, L. Aravind, and Joshua A. Arribere. 2020. "NONU-1 Encodes a Conserved Endonuclease Required for MRNA Translation Surveillance." Cell Reports 30 (13): 4321-4331.e4.

"GSEApy." n.d. https://github.com/zqfang/gseapy.

Guydosh, Nicholas R., and Rachel Green. 2014. "Dom34 Rescues Ribosomes in 3' Untranslated Regions." Cell 156 (5): 950-62. 
Hendrick, J. L., P. G. Wilson, I. I. Edelman, M. G. Sandbaken, D. Ursic, and M. R. Culbertson. 2001. "Yeast Frameshift Suppressor Mutations in the Genes Coding for Transcription Factor Mbf1p and Ribosomal Protein S3: Evidence for Autoregulation of S3 Synthesis." Genetics 157 (3): 1141-58.

Hickey, Kelsey L., Kimberley Dickson, J. Zachery Cogan, Joseph M. Replogle, Michael Schoof, Karole N. D'Orazio, Niladri K. Sinha, et al. 2020. "GIGYF2 and 4EHP Inhibit Translation Initiation of Defective Messenger RNAs to Assist Ribosome-Associated Quality Control." Molecular Cell 79 (6): 950962.e6.

Ikeuchi, Ken, Toshiaki Izawa, and Toshifumi Inada. 2019. "Recent Progress on the Molecular Mechanism of Quality Controls Induced by Ribosome Stalling." Frontiers in Genetics. https://doi.org/10.3389/fgene.2018.00743.

Inada, Toshifumi. 2017. "The Ribosome as a Platform for MRNA and Nascent Polypeptide Quality Control." Trends in Biochemical Sciences 42 (1): 5-15.

Ingolia, Nicholas T., Sina Ghaemmaghami, John R. S. Newman, and Jonathan S. Weissman. 2009. "Genome-Wide Analysis in Vivo of Translation with Nucleotide Resolution Using Ribosome Profiling." Science 324 (5924): 218-23.

Ishimura, Ryuta, Gabor Nagy, Ivan Dotu, Huihao Zhou, Xiang-Lei Yang, Paul Schimmel, Satoru Senju, Yasuharu Nishimura, Jeffrey H. Chuang, and Susan L. Ackerman. 2014. "RNA Function. Ribosome Stalling Induced by Mutation of a CNS-Specific TRNA Causes Neurodegeneration." Science 345 (6195): 455-59.

Juszkiewicz, Szymon, Viswanathan Chandrasekaran, Zhewang Lin, Sebastian Kraatz, V. Ramakrishnan, and Ramanujan S. Hegde. 2018. "ZNF598 is a Quality Control Sensor of Collided Ribosomes." Molecular Cell 72 (3): 469-481.e7.

Juszkiewicz, Szymon, Greg Slodkowicz, Zhewang Lin, Paula Freire-Pritchett, Sew-Yeu Peak-Chew, and Ramanujan S. Hegde. 2020. "Ribosome Collisions Trigger Cis-Acting Feedback Inhibition of Translation Initiation." ELife. https://doi.org/10.7554/elife.60038.

Koutmou, Kristin S., Anthony P. Schuller, Julie L. Brunelle, Aditya Radhakrishnan, Sergej Djuranovic, and Rachel Green. 2015. "Ribosomes Slide on Lysine-Encoding Homopolymeric A Stretches." ELife 4 (February). https://doi.org/10.7554/eLife.05534.

Kuroha, Kazushige, Mayuko Akamatsu, Lyudmila Dimitrova, Takehiko Ito, Yuki Kato, Katsuhiko Shirahige, and Toshifumi Inada. 2010. "Receptor for Activated C Kinase 1 Stimulates Nascent PolypeptideDependent Translation Arrest." EMBO Reports 11 (12): 956-61.

Letzring, Daniel P., Kimberly M. Dean, and Elizabeth J. Grayhack. 2010. "Control of Translation Efficiency in Yeast by Codon-Anticodon Interactions." RNA (New York, N.Y.) 16 (12): 2516-28.

Letzring, Daniel P., Andrew S. Wolf, Christina E. Brule, and Elizabeth J. Grayhack. 2013. "Translation of CGA Codon Repeats in Yeast Involves Quality Control Components and Ribosomal Protein L1." RNA (New York, N.Y.) 19 (9): 1208-17.

Martin, Paige B., Yu Kigoshi-Tansho, Roger B. Sher, Gianina Ravenscroft, Jennifer E. Stauffer, Rajesh Kumar, Ryo Yonashiro, et al. 2020. "NEMF Mutations That Impair Ribosome-Associated Quality Control Are Associated with Neuromuscular Disease." Nature Communications 11 (1): 4625.

Matsuo, Yoshitaka, Ken Ikeuchi, Yasushi Saeki, Shintaro Iwasaki, Christian Schmidt, Tsuyoshi Udagawa, Fumiya Sato, et al. 2017. "Ubiquitination of Stalled Ribosome Triggers Ribosome-Associated Quality Control." Nature Communications 8 (1). https://doi.org/10.1038/s41467-017-00188-1.

McCusker, John H. 2017. "Introducing MX Cassettes into Saccharomyces Cerevisiae." Cold Spring Harbor Protocols 2017 (4): db.prot088104.

McGlincy, Nicholas J., and Nicholas T. Ingolia. 2017. "Transcriptome-Wide Measurement of Translation by Ribosome Profiling." Methods (San Diego, Calif.) 126 (August): 112-29.

Meydan, Sezen, and Nicholas R. Guydosh. 2020. "Disome and Trisome Profiling Reveal Genome-Wide Targets of Ribosome Quality Control." Molecular Cell 79 (4): 588-602.e6. 
Mishima, Yuichiro, and Yukihide Tomari. 2016. "Codon Usage and 3' UTR Length Determine Maternal MRNA Stability in Zebrafish." Molecular Cell 61 (6): 874-85.

Morita, M., L. W. Ler, M. R. Fabian, N. Siddiqui, M. Mullin, V. C. Henderson, T. Alain, et al. 2012. "A Novel 4EHP-GIGYF2 Translational Repressor Complex Is Essential for Mammalian Development." Molecular and Cellular Biology. https://doi.org/10.1128/mcb.00455-12.

Muhlrad, D., C. J. Decker, and R. Parker. 1994. "Deadenylation of the Unstable MRNA Encoded by the Yeast MFA2 Gene Leads to Decapping Followed by 5'-->3' Digestion of the Transcript." Genes \& Development 8 (7): 855-66.

Notredame, C., D. G. Higgins, and J. Heringa. 2000. "T-Coffee: A Novel Method for Fast and Accurate Multiple Sequence Alignment." Journal of Molecular Biology 302 (1): 205-17.

O'Connor, Patrick B. F., Dmitry E. Andreev, and Pavel V. Baranov. 2016. "Comparative Survey of the Relative Impact of MRNA Features on Local Ribosome Profiling Read Density." Nature Communications 7 (October): 12915.

Opitz, Nadine, Kerstin Schmitt, Verena Hofer-Pretz, Bettina Neumann, Heike Krebber, Gerhard H. Braus, and Oliver Valerius. 2017. "Capturing the Asc1p/Receptor for Activated C Kinase 1 (RACK1) Microenvironment at the Head Region of the 40S Ribosome with Quantitative BiolD in Yeast." Molecular \& Cellular Proteomics: MCP 16 (12): 2199-2218.

Pelechano, Vicent, Wu Wei, and Lars M. Steinmetz. 2015. "Widespread Co-Translational RNA Decay Reveals Ribosome Dynamics." Cell 161 (6): 1400-1412.

Perez-Riverol, Yasset, Attila Csordas, Jingwen Bai, Manuel Bernal-Llinares, Suresh Hewapathirana, Deepti J. Kundu, Avinash Inuganti, et al. 2019. "The PRIDE Database and Related Tools and Resources in 2019: Improving Support for Quantification Data." Nucleic Acids Research 47 (D1): D442-50.

Peter, Daniel, Vincenzo Ruscica, Praveen Bawankar, Ramona Weber, Sigrun Helms, Eugene Valkov, Cátia Igreja, and Elisa Izaurralde. 2019. "Molecular Basis for GIGYF-Me31B Complex Assembly in 4EHPMediated Translational Repression." Genes \& Development 33 (19-20): 1355-60.

Pochopien, Agnieszka A., Bertrand Beckert, Sergo Kasvandik, Otto Berninghausen, Roland Beckmann, Tanel Tenson, and Daniel N. Wilson. 2021. "Structure of Gcn1 Bound to Stalled and Colliding $80 \mathrm{~S}$ Ribosomes." Proceedings of the National Academy of Sciences of the United States of America 118 (14): e2022756118.

Presnyak, Vladimir, Najwa Alhusaini, Ying-Hsin Chen, Sophie Martin, Nathan Morris, Nicholas Kline, Sara Olson, et al. 2015. "Codon Optimality Is a Major Determinant of MRNA Stability." Cell 160 (6): 1111-24.

Radhakrishnan, Aditya, Ying-Hsin Chen, Sophie Martin, Najwa Alhusaini, Rachel Green, and Jeff Coller. 2016. "The DEAD-Box Protein Dhh1p Couples MRNA Decay and Translation by Monitoring Codon Optimality." Cell 167 (1): 122-132.e9.

Robert, Xavier, and Patrice Gouet. 2014. "Deciphering Key Features in Protein Structures with the New ENDscript Server." Nucleic Acids Research 42 (Web Server issue): W320-4.

Saeed, A. I., V. Sharov, J. White, J. Li, W. Liang, N. Bhagabati, J. Braisted, et al. 2003. "TM4: A Free, OpenSource System for Microarray Data Management and Analysis." BioTechniques 34 (2): 374-78.

Saito, Kazuki, Wataru Horikawa, and Koichi Ito. 2015. "Inhibiting K63 Polyubiquitination Abolishes No-Go Type Stalled Translation Surveillance in Saccharomyces Cerevisiae." PLoS Genetics 11 (4): e1005197.

Sezen, Bengü, Matthias Seedorf, and Elmar Schiebel. 2009. "The SESA Network Links Duplication of the Yeast Centrosome with the Protein Translation Machinery." Genes \& Development 23 (13): 155970.

Sharma, Pamila, Fu Yan, Victoria A. Doronina, Helena Escuin-Ordinas, Martin D. Ryan, and Jeremy D. Brown. 2012. "2A Peptides Provide Distinct Solutions to Driving Stop-Carry on Translational Recoding." Nucleic Acids Research 40 (7): 3143-51. 
Simms, Carrie L., Liewei L. Yan, Jessica K. Qiu, and Hani S. Zaher. 2019. "Ribosome Collisions Result in +1 Frameshifting in the Absence of No-Go Decay." Cell Reports 28 (7): 1679-1689.e4.

Sinha, Niladri K., Alban Ordureau, Katharina Best, James A. Saba, Boris Zinshteyn, Elayanambi Sundaramoorthy, Amit Fulzele, et al. 2020. "EDF1 Coordinates Cellular Responses to Ribosome Collisions." ELife 9 (August). https://doi.org/10.7554/eLife.58828.

Sitron, Cole S., Joseph H. Park, and Onn Brandman. 2017. "Asc1, Hel2, and Slh1 Couple Translation Arrest to Nascent Chain Degradation." RNA 23 (5): 798-810.

Subramanian, Aravind, Pablo Tamayo, Vamsi K. Mootha, Sayan Mukherjee, Benjamin L. Ebert, Michael A. Gillette, Amanda Paulovich, et al. 2005. "Gene Set Enrichment Analysis: A Knowledge-Based Approach for Interpreting Genome-Wide Expression Profiles." Proceedings of the National Academy of Sciences of the United States of America 102 (43): 15545-50.

Sundaramoorthy, Elayanambi, Marilyn Leonard, Raymond Mak, Jeffrey Liao, Amitkumar Fulzele, and Eric J. Bennett. 2017. "ZNF598 and RACK1 Regulate Mammalian Ribosome-Associated Quality Control Function by Mediating Regulatory 40S Ribosomal Ubiquitylation." Molecular Cell 65 (4): 751760.e4.

Sweet, Thomas, Carrie Kovalak, and Jeff Coller. 2012. "The DEAD-Box Protein Dhh1 Promotes Decapping by Slowing Ribosome Movement." PLoS Biology 10 (6): e1001342.

Tesina, Petr, Laura N. Lessen, Robert Buschauer, Jingdong Cheng, Colin Chih-Chien Wu, Otto Berninghausen, Allen R. Buskirk, Thomas Becker, Roland Beckmann, and Rachel Green. 2020. "Molecular Mechanism of Translational Stalling by Inhibitory Codon Combinations and Poly(A) Tracts." The EMBO Journal 39 (3): e103365.

Tsuboi, Tatsuhisa, Kazushige Kuroha, Kazuhei Kudo, Shiho Makino, Eri Inoue, Isao Kashima, and Toshifumi Inada. 2012. "Dom34:Hbs1 Plays a General Role in Quality-Control Systems by Dissociation of a Stalled Ribosome at the 3' End of Aberrant MRNA." Molecular Cell 46 (4): 518-29.

Tyanova, Stefka, Tikira Temu, and Juergen Cox. 2016. "The MaxQuant Computational Platform for Mass Spectrometry-Based Shotgun Proteomics." Nature Protocols 11 (12): 2301-19.

Wagih, Omar, Matej Usaj, Anastasia Baryshnikova, Benjamin VanderSluis, Elena Kuzmin, Michael Costanzo, Chad L. Myers, Brenda J. Andrews, Charles M. Boone, and Leopold Parts. 2013. "SGAtools: One-Stop Analysis and Visualization of Array-Based Genetic Interaction Screens." Nucleic Acids Research 41 (Web Server issue): W591-6.

Wang, Jiyu, Jie Zhou, Qidi Yang, and Elizabeth J. Grayhack. 2018. "Multi-Protein Bridging Factor 1(Mbf1), Rps3 and Asc1 Prevent Stalled Ribosomes from Frameshifting." ELife 7 (November). https://doi.org/10.7554/eLife.39637.

Weber, Ramona, Min-Yi Chung, Csilla Keskeny, Ulrike Zinnall, Markus Landthaler, Eugene Valkov, Elisa Izaurralde, and Cátia Igreja. 2020. "4EHP and GIGYF1/2 Mediate Translation-Coupled Messenger RNA Decay." Cell Reports 33 (2): 108262.

Webster, Michael W., Ying-Hsin Chen, James A. W. Stowell, Najwa Alhusaini, Thomas Sweet, Brenton R. Graveley, Jeff Coller, and Lori A. Passmore. 2018. "MRNA Deadenylation Is Coupled to Translation Rates by the Differential Activities of Ccr4-Not Nucleases." Molecular Cell 70 (6): 1089-1100.e8.

Wu, Colin Chih-Chien, Amy Peterson, Boris Zinshteyn, Sergi Regot, and Rachel Green. 2020. "Ribosome Collisions Trigger General Stress Responses to Regulate Cell Fate." Cell 182 (2): 404-416.e14.

Wu, Colin Chih-Chien, Boris Zinshteyn, Karen A. Wehner, and Rachel Green. 2019. "High-Resolution Ribosome Profiling Defines Discrete Ribosome Elongation States and Translational Regulation during Cellular Stress." Molecular Cell 73 (5): 959-970.e5.

Wu, Qiushuang, Santiago Gerardo Medina, Gopal Kushawah, Michelle Lynn DeVore, Luciana A. Castellano, Jacqelyn M. Hand, Matthew Wright, and Ariel Alejandro Bazzini. 2019. "Translation Affects MRNA Stability in a Codon-Dependent Manner in Human Cells." ELife 8 (April). https://doi.org/10.7554/eLife.45396. 
Xie, Zhuorui, Allison Bailey, Maxim V. Kuleshov, Daniel J. B. Clarke, John E. Evangelista, Sherry L. Jenkins, Alexander Lachmann, et al. 2021. "Gene Set Knowledge Discovery with Enrichr." Current Protocols 1 (3): e90.

Yan, Liewei L., Carrie L. Simms, Fionn McLoughlin, Richard D. Vierstra, and Hani S. Zaher. 2019. “Oxidation and Alkylation Stresses Activate Ribosome-Quality Control." Nature Communications 10 (1): 5611. Yan, Liewei L., and Hani S. Zaher. 2019. "How Do Cells Cope with RNA Damage and Its Consequences?" The Journal of Biological Chemistry 294 (41): 15158-71.

\section{Data Availability}

Ribo-seq data is available in the NCBI Gene Expression Omnibus (GEO) (https://www.ncbi.nlm.nih.gov/geo/) database with the accession GSE189404. The mass spectrometry proteomics data have been deposited to the ProteomeXchange Consortium via the PRIDE (Perez-Riverol et al. 2019) partner repository with the dataset identifier PXD030076.

\section{Acknowledgements}

We thank Allen R. Buskirk, Niladri Sinha, and Nicolle Rosa Mercado for careful reading of the manuscript and all Green lab members for helpful discussions throughout this study. High throughput sequencing was performed at the Johns Hopkins Genetic Resources Core Facility (RRID: SCR_018669) and the Johns Hopkins Single Cell and Transcriptomics Core. Mass spectrometry was performed by the Johns Hopkins Mass Spectrometry Core.

\section{Funding}

\section{Canadian Institutes of Health Research Foundation Grant FDN-159913}

- Grant W. Brown

\section{National Institutes of Health (R37GM059425)}

- Rachel Green 
bioRxiv preprint doi: https://doi.org/10.1101/2021.12.01.470814; this version posted December 1, 2021. The copyright holder for this preprint (which was not certified by peer review) is the author/funder, who has granted bioRxiv a license to display the preprint in perpetuity. It is made available under aCC-BY-NC-ND 4.0 International license.

\section{National Institutes of Health (5T32GM135131-02)}

